\title{
A Culturally Competent Phenotypic Evaluation/Obesity As- sessment in African and African American Populations: Pilot Study
}

\author{
Catrina Johnson ${ }^{1,2,3^{*}}$, Robert Corruccini ${ }^{4}$, Daniel Becque ${ }^{5,6}$, Wanki Moon ${ }^{7}$, Kola Ajuwon ${ }^{8}$, and \\ David Lightfoot ${ }^{1,2}$
}

${ }^{1}$ Genomics Core Facility, Southern Illinois University Carbondale, IL 62901,USA; ${ }^{2}$ Department of Plant, Soil \& Ag Systems, Southern Illinois University Carbondale, IL 62901, USA; ${ }^{3}$ Center for Health, Nutrition \& Biomedicine P.O. Box 1062, Park Forest, IL 60466;USA; ${ }^{4}$ Department of Anthropology, Southern Illinois University Carbondale, IL 62901, USA; ${ }^{5}$ Department of Kinesiology, Southern Illinois University Carbondale, IL 62901, USA; ${ }^{6}$ Department of Physiology, Southern Illinois University Carbondale, IL 62901, USA; ${ }^{7}$ Department of Agribusiness,Southern Illinois University Carbondale, IL 62901, USA; 8 Department of Animal Sciences \& Nutrition, Purdue University-Elkhart, IN 49707, USA.

Received: January 16, 2019 / Accepted: June 23, 2019

\section{Abstract}

Best practice, movement towards individualized medicine and deployment of effective models that impact the diabetes epidemic and its related precursors like insulin resistance and the metabolic syndrome, requires terminal use of BMI, a biologically meaningless and crude indicator of obesity, in favor of effective and culturally-competent non-relative body composition evaluation of genetically determined adiposity, that untenably compares values among groups. African Americans are among the increasingly affected groups for diabetes and possess unique composition variation requiring proper intra-cultural evaluation independent of inter-ethnic Eurocentric assumptions that over assesses obesity risk. Incorporating use of $4 \mathrm{C}$ models to evaluate adiposity and assess risk for diabetic predisposition and onset, provides an effective, unbiased assessment of the cultural components inherent within body composition variation among ethnicity, age and gender. Obesity and type 2 diabetes onset and pre-disposition was assessed phenotypically, in creation of a body mass profile among African and African American groups, using 4C model, photography, anthropometry, somatotype and genetic evaluation. Environmental, obeseogenic cultural factors were also explored. BMI was not found to be an accurate predictor of adiposity in Africans and African Americans. West Africans and other African Americans were found to be an accurate and cultur

ally competent reference population for African American physiology vs. European. Africans and African Americans were found to be heavier and less fat and normal weight at higher BMI, attributable to cultural acceptance and more fat free mass. Skeletal weights were heavy (6-7lbs) among Africans and African Americans. African Americans had heavier bone density than Africans but African bone weight increased the longer they stayed in the U.S. BMI falsely assumed the presence of fat in this population. $70 \%$ of body mass was attributable to muscle, confirming the mesomorphic phenotype in these groups. African American women were larger than their male counterparts vs. Africans, a sexual dimorphic indicator that may be attributable to the absence of incarcerated Black male phenotypic data in this study.

Keywords: African-Americans, West-Africans, physiology, obesity, BMI.

*Corresponding author: johnsoncatrina@gmail.com

This is an Open Access article distributed under the terms of the Creative Commons Attribution License (http://creativecommons.org/licenses/by/3.0/), which permits unrestricted use, distribution, and reproduction in any medium, provided the original work is properly cited. 


\section{Introduction}

\section{Phenotypic Evaluation/Obesity Assessment}

BMI, a ratio of weight over height, is a key tool used to measure obesity and diabetic predisposition by clinicians and government measurement standards like the NHANES survey. It emanated from 1959 ideal weight tables generated by Metropolitan Life Insurance Company that was exclusive to most of the population relative to White, upper class males (Loos et al., 2008) and became a mandated shibboleth as a proper measure of adiposity by researchers and publishers by the 1985 NIH Consensus Panel (Kuczymarksi and Flegal, 2000). What is problematic about this acceptance, is that a standard that fails to represent a proper relative sample or that excludes groups, cannot be used as a universal measure for all groups (Harrison, 1985). And the notion of "ideal weight" has been found to be biased and inaccurate as a universal standard (Knapp, 1983) across ethnicity, class, gender and age. Adipose tissue contains hormones that can upset metabolic homestasis with regards to an increase in insulin release. However, it is not the general bodily presence of adipose tissue that increases susceptibility to diabetes, rather, the location specific adiposity(Bjorntorp, 1985). Fat patterning is not relatively assumed but genetically determined (Wagner and Heyward, 2000). Adipose tissue located around the waist is correlated with metabolic syndrome, glucose intolerance-a precursor to diabetes, and diabetes onset (Bjorntorp, 1985; Fox, 2008). Body composition is an acceptable and proven method of evaluation of adiposity, and ideal weight (Wagner and Heyward, 2000) and has a variation across cultures (Harrison, 1985). African Americans have been shown to have a higher percentage of lean, fat free masses that include heavier skeletal weights, muscle and bone mineral content (BMC), longer extremities, adipose concentrations in the trunk, subscapular, back and lateral areas and low waist to hip ratios (Schutte et al., 1984; Hortobagyi et al., 1990). The universal body composition model that evaluates body fat percentage, historically was exclusive to African Americans relative to the evaluation of White, male cadavers and is therefore not an accurate representation for adiposity in African Americans. The relationship between adiposity and weight is weakly correlated (Harrison, 1985).Adipose tissue, accepted to be the result of energy storage over expenditure, is not correlated with obesity in Africans and African Americans' tissue (Ebersole et al., 2008). Body Mass Index represents an assumption of adiposity and its equal distribution and has a strong cultural component (Kleerokoper et al., 1994) that is different among ethnicity, culture, gender and age (Gallagher et al. 1996). BMI is not a useful tool to evaluate adiposity (Kaarma et al., 2009; Kennedy et al., 2009; Smalley et al., 1990) among ethnicities. It is not comparable across ethnic groups (Satija, 2016). It has been shown to over-estimate obesity among African Americans (Aloia et al., 1997; Aloia et al., 1998). The $4 \mathrm{C}$ model is a proper tool to evaluate body fat that eliminates bias across ethnicity (Mott and et al., 1999; Durenberg and Durenberg, 2001).

\section{Genes and Variants Associated with Obesity}

Obesity has a genetic component. The following genes have been affiliated with obesity: 23HNF4A haplotypes in Intron 37 region,23HNF4A haplotypes in $\mathrm{P} 2$ promoter region, ADRB2, ADRB3, AGRP, ANKRD26, APOE (SNPs), CART, CART (mutation), CDKAL1 chromosome 6 SNP-rs9350270, Chromosome 3p26, Chromosome 6, E2F3 chromosome-6 SNP rs6939190, E2F3 chromosome 6 SNP-rs6939190, ENPP1, ENPP1 (3 allele haplotype), ESR 1 absence or variation, FETUB, FTO, FTO (SNP-rS11219800, FTO intron 1, GHRL, GNPA2， GNPDA2 (SNP-rs10938397), IL-6, IQGAP1, KCTD, MC4R, MC4R (mutation), MC4R (SNP s17782313), MC4R (SNP rs12970134), MC4R (SNP-rs17782313), MC4R mutation, MC4R(SNP- rs12970134), MC5R, MTCH2, MTCH2 (SNP-rs4752856), Multiple rare deleterous variants, MYO18B, MYO18B, NEGR1, NEGR1 (SNP-rs2815752), NR0B2, NROB2 (mutation), PCSKI (variation in), PDSS2, POMC, POMC (mutation), PPARG, PPARGC1B, PTPRD, PYY, RELA (1KBKB variants), rs2241766(adiponectin), rs23047595, rs2304795, rs2304795, rs2745367(resistin), rs8179071, rs894160, SDC3, SDC3 (SNPs), SH2B1, SH2B1 (SNP-rs7498665), SIM1, SNP-rs6004901, SNP-rs6870962, SSTR2, TMEM18, UCP1, UCP3 (Ahituv et al., 2007; Bagwell et al., 2005; Barroso, 2005; Bouchard et al., 1990; Branson et al., 2003; Calton and Vaisse, 2009; Chambers et al., 2008; Dong et al., 2003; Dong et al., 2005; Doumatey, 2009; Dubern et al., 2001; Farooqi et al., 2003; Gallagher et al., 2007; Lucas et al., 2011); Meyre et al., 2009; Norman et al., 1997; Paganini-Hill et al., 1981; Proctor, 2009; Sutton et al., 2005; Willer et al., 2009; Wing, 2010; Zonta et al., 1987; Nishigori et al., 2001).

\section{Obesity Genotypes Affiliated with African Americans}

Hassanein et al. (2010) discovered an association between variants rs3751812 and rs9941349 with BMI in African Americans. Wing et al. (2010) found that genetic heterogeneity between African Americans, Hispanic Americans and Caucasian Americans was affiliated with FTO intron 1. He also found the ratio of visceral to subcutaneous fat (VSR) to be associated with MYO18B, PDSS2 and IQGAP1. Doumatey et al. (2009) found the IL-6 was associated to body mass index (BMI), waist hip ratio (WHR), and the homeostatic model assessment (HOMA IR) for insulin resistance in African Americans. The environmental mode of action being telomere shortening (Epel et al., 2004). It affects IL-6 (Lin et al., 2012). Doumatey et al. (2009) also found rs2241766 (adiponectin) to be associated with waist hip ratios and rs2745367(resistin) associated with circulating resistin in African Americans and West Africans. These groups were also found to regulate adopokines differently. Proctor et al. (2009) associated subcutaneous adipose tissue in African Americans with E2F3 chromosome 6-SNP rs4710930 and rs6939190.

Bagwell et al. (2005) found that 23HNF4A haplotypes in the P2 promoter region and 3-intron 7 region related significantly to measures of obesity in Hispanics and African Americans. Barroso et al, 2005 found association with risk-raising waist 
circumference, waist-hip-ratios, estimated percent body fat, body weight, and BMI $>35$ to be associated with gene variants rs2304795, rs894160 and rs230475. Metabolic syndrome prevalence was associated with rs2304794.Gallagher et al, 2004 associated the OPRM1 gene to type 2 diabetes susceptibility among African Americans. And Sutton et al. (2005) discovered a link between chromosome 3p26 and obesity phenotypes in African Americans.

\section{Body Mass Index (BMI)}

Is diabetes- as predicted by Body Mass Index (BMI) alone amongst African Americans- a problematic assessment? BMI is a key tool used to measure obesity and diabetic predisposition that uses the standards of Americans of European descent as a normative. BMI is defined as: a ratio of weight over height squared. It is widely accepted as a modernized height and weight tables that standardizes the measure of body fat using a weight over height ratio.

\section{BMI and Ethnicity}

There is variation in BMI among ethnicity. For the same BMI Black women have $1 \%$ less body fat that White women (Evans et al., 2006). Currently accepted BMI, when adjusted for race, produced a low sensitivity to fatness (Evans et al., 2006). It is different among Whites and Blacks (Evans et al., 2006) and is an imprecise measure of fatness across ethnicity (Mills et al., 2007). At the same BMI African American men had lower visceral/belly fat than White and Hispanic men (Carroll et al., 2008) and controlled for age, had less truncal fat and more skeletal muscle than Hispanic men (Aleman-Mateo et al., 2009).BMI cut off points are necessary to determine metabolic risk among different ethnic groups (Carroll et al., 2008). Environmental factors like screen time, school commuting and consumption of calorie dense snacks and sweetened drinks is dependent upon BMI and ethnicity (Singh et al., 2009).

\section{BMI and Environmental Factors}

In a study of African Americans and West Africans, BMI in African Americans was associated with insulin resistance (Doumatey, 2009).Among middle aged and older women, weight gain was affiliated with age (Ortega-Alonso et al., 2009).Variation in ponderosity-body weight relative to height as determined by BMI-23\% was attributed to environmental or non-genetic factors (Komlos et al., 2009). 48\% of variation among RFPI, skinfold thickness was due to environmental effects (Hasstedt et al., 1989). At all levels of BMI only $10 \%$ of fat cells die and are renewed annually. Adult fat cells are established during the childhood environment (Spalding, 2008). Disadvantaged community has an effect on BMI. It reduces racial disparities in BMI but does not affect BMI over time (Ruel et al., 2010). Physical activity can diminish the additive effects of the heritability of BMI (Mustelin et al., 2009). Chronic stress increases cortisol levels and BMI among populations with no college degree (Daniel et al., 2006). Cultural differences in BMI may be explained by behaviors effecting energy expenditure like television viewing, commuting to school and consumption of fruit juices (Singh et al., 2009). BMI was associated with smoking in AA males.

\section{BMI and Inheritance}

A child with one or more parents overweight, inherits an increase risk for overweight (Danielzik et al., 2002).The BMI of parents affect the offspring (Li et al., 2009; Robl et al., 2008). Heritability of BMI from parents was found to be $79 \%$. Among males, physical activity reduced waist circumference and heritability to $78 \%$ and females reduced to $56 \%$ and $71 \%$ with physical activity (Mustelin et al., 2009). In an international study BMI heritability was measured at $80-82 \%$ (Hjelmborg et al., 2008). In an international study of 7 and $10 \mathrm{yr}$ olds, BMI heritability was found at $60-74 \%$. The same environmental and genetic factors responsible for variation in BMI caused obesity (Haworth et al., 2008). A study by Hunt et al $(n=38,759)$ found evidence that increases in BMI act upon the genotype, increasing the allele frequency of the FTO gene (Hunt et al., 2008). In Dutch families weight class (thin, median, overweight or obese) and BMI are inherited from mothers and fathers. Different variants effected change and BMI levels. Genetic influences related to BMI levels is $60 \%$. Genetic influences related to BMI change is $64 \%$. (Ortega-Alonso et al., 2009). Dong et al. (2005) located chromosomes responsible for genomic imprinting of obesity from parents among Europeans. BMI imprinting from the father was on 12Q24 and on 10p12. The additive and non-additive genetic effects in African Americans on BMI are different from European Americans (Duncan et al., 2009). A small scale twins study $(\mathrm{N}=12)$ revealed genetic factors affecting the body's tendency to store energy as fat or lean tissue and various determinants of resting energy expenditure. This tendency affected regional fat distribution and abdominal/visceral fat. Hasstedt et al., in a study of 774 adults discovered $42.3 \%$ of variation in the relative fat pattern index (RFPI)-a ratio of subscapular skinfold thickness to the sum of subscapular and suprailac skinfold thickness, was due to recessive allele inheritance. $9.5 \%$ was polygenic and $48.2 \%$ attributable to random environmental effects(Hasstedt et al. 1989). Weight class (thin, median weight, overweight or obese) was found to be strongly related to the BMI of the mother $(\mathrm{p}=.0001)$ and the father $(\mathrm{p}=.02)$.

$\mathrm{BMI}$ is not a valid indicator of regional fat distribution (Kok et al., 2004)."Controlling for bone size, there is considerable variation in density and thus weight of the skeleton in human adults and this variation is correlated with age, sex and race (Harrison, 1985)." -Bone density is associated with hormonal regulation. Leptin levels have an inverse relationship to the regulation of ERa signaling. Increased levels of leptin in animal models was shown to increase bone density (Ohlsson, 2000)."An ideal weight cannot be identified at a point in time for a person or person differing from the group or groups on which the table was based (Harrison, 1985)." It is not accurate across ethnicity (Evans et al., 2006)"; (Mills et al., 2007). Many studies have disproven it as a reliable measure of adipoisity. 


\section{BMI Not a Useful Measure}

BMI cutoffs are not accurate across ethnicity (Evans, 2006; Mills, 2007). It is insensitive to the variation in body composition across ethnicity (Kok, 2004). When compared with measures of skinfolds and body composition, BMI does not correlate with body fat, height or length of extremities (Kaarma et al., 2009) and is inaccurate across levels of fatness (Freedman and Sherry, 2009). BMI is not a biological indicator of body fat distribution (Kok,,2004) and should not be used to evaluate obesity prevalence (McAdams et al., 2007). It introduces bias and misclassification (Rothman, 2008), overpredicts overweight and underpredicts obesity and should not be used in scientific or clinical research (Kennedy et al., 2009). BMI is a poor predictor of fat mass in adolescents where FFM is attributed to variation in BMI (Freedman et al., 2005).Using DEXA, BMI was found to be a measure of weight and not fatness or adiposity (Freedman and Sherry, 2009). Height and weight as absolute values cannot be expressed by BMI because it represents part and not the whole body (Kaarma et al., 2009). In meta-analysis it underpredicts excess body fat in half of its study participants(Okorodudu et al., 2010). Why is it still being used?

Given this history of quasi-breeding and phenotypic selection caused by slavery and its eight generations of African American commoditization, would the descendants of slaves thusly affected, present a BMI within the same normative range of a European culture that experienced no equivalent episodes of selection? Could African Americans posses a genetic tendency towards a larger BMI in response to historic selection pressures practiced in the era of slavery? This question is of general cultural significance and is an essential prerequisite towards validation of the "epidemic" of obesity/diabetes amongst Blacks.

\section{African American Physiology}

\section{Body Composition and Genetics}

Waist circumference is inherited via parental BMI (Mustelin et al., 2009). Genes determine body fat percentage and leanness (Ahituv et al., 2007).

\section{Body Composition And Ethnicity}

Body composition is variable across, ethnicity, age and sex and must be adjusted accordingly to determine health risks due to fatness (Kok et al., 2004). Race adds to prediction of body fat. For the same BMI Black women have 1\% less body fat that White women (Evans et al., 2006). Percent body fat is different between Black and White (Evans et al., 2006). Caucasian males have higher body fat than African American men (Mills et al., 2007). African American fat increases with age faster than Asians and Hispanics (Mills et al., 2007). White women have higher percent body fat than other races (Mills, 2007). At low BMI Asian women have the highest percent body fat (Mills et al., 2007). At the same BMI and waist circumference, African
American men had lower visceral fat than White and Hispanic men (Carroll et al., 2008). Whites and Hispanics have more visceral fat than African American women (Carroll et al., 2008). Visceral fat (adipose) tissue defines metabolic risk in different populations (Carroll et al., 2008). Different waist circumference (WC) and BMI cut off points are necessary to determine metabolic risk among different ethnic groups (Carroll et al., 2008). At the same BMI and age, Mexicans have more truncal (derriere) fat and less total appendicular skeletal muscle than African Americans (Aleman-Mateo et al., 2009).

\section{Body Composition and Environment}

Using a micro environmental analysis of phenotype shows that waist circumference inherited via parental BMI is subject to reduction by physical exercise (Mustelin et al., 2009). A Macro phenotypic analysis using Environmental Systems Theory reveals that hot climates encourage tall and lean phenoytpes (Walker and Hamilton, 2008).Cold climates encourage short and round phenotypes (Walker and Hamilton, 2008). Dense populations (i.e. Asia, India) encourage petite phenotypes via natural selection and small population density favors the large phenotype (Walker and Hamilton, 2008). Genes and mutations have been associated with obese phenotypes of geographical regions of North America (Feitosa et al., 2002), Europe (Bagwell et al., 2005; Branson et al., 2003), Japan (Chambers et al., 2008), Italy (Dubern et al., 2001) and cultures like the Pima Indians (Farooqi et al., 2003).

\section{Genetics}

Genes Associated with BMI in the literature included: BMIQ1, 7q31; BMIQI, BMIQI (near leptin gene) , 7q32.3;BMIQ2, 13q14; BMIQ3, 6q23-q25; BMIQ4, (variation in UCP2), 11q24; BMIQ5, 16p13; BMIQ6, 20pterp11.2; BMIQ7, 4p15-p14; BMIQ8, 10p; BMIQ9, (variation in MC3R), 20q; BMIQ10; BMIQ 11; BMIQ12, (variation in PCSK1); BMIQ13; BMIQ14; BMIQ15, (PRKCA); BMIQ16; PPARG2 (polymorphism); NPC1; ADIPOQ, 10q; Xq24; 5q15q21; 2q14.1; 16q12.2; 17q23.2-q25.1; 16p11.2;18q11;3q27; MTMR9; NPCI (rs1805081); MAF (rs1424233); GPRC5B (proximity variant); MC4R (susceptibility loci); POMC; SH2B1; BDNF; FTO; IRS1; SPRY2; MC4R (rs17782313)Higher BMI; MC4R (rs17782313); MC4R (rs17782313-C). Increased risk of type 2 diabetes; MC4R (variant); FTO; HTR1B; HTR1B; HTR1B; UCP2; UCP3; VDR; IGF1; IL6R; GHSR; PPARGC1A LEP; CYP19A1; GLDN; HTR1B (polymorphism); CYP19A1 (polymorphism); HTR1B (polymorphism); HTR2C (AA, BMI X Environment); ADIPOR1 (AA, BMI $x$ Environment); IGFBP3 (CA, BMI x environment); ADIPOR (CA, BMI x environment); PPARG (CA, BMI x environment); PPARG (CA, BMI x environment), 8p23-p22; 18q22-q23; 16q22-q23; 16p12; rs243650 (effecting allele T); rs534870 (effecting allele A); rs17782313; C allele of rs17782313; C allele of rs17782313; rs12970134; 6 (rs13212041; 6 (rs6296); 6 (rs4140535); 11 (rs17132534); 11 (rs7110607); 12 (rs4334089); 12 (rs6214); 18rs (17066829); 1 (rs12083537); 1 (rs12083537); 
3 (rs11929140); 3 (rs2948694); 4 (rs6821591); 7 (rs2278815); 15 (rs1902584); 15 (rs1961177); (rs4140535) for BMI <25, 2529, 30-34, >35)); rs1902584 for BMI, 25, 25-29, 30-34, >35); rs4140535 for BMI $<25 \mathrm{~m}$ 25-29, 30-34, $>35$ ); rs 1902584 for $\mathrm{BMI}<25,25-29,30-34,>35)$; $\mathrm{X}$ (rs17095676xcigarette smoking) $\mathrm{P}=.001 ; 1$ (rs6672643xcigarette smoking (current) $\mathrm{P}=.001$; 7 (rs6670xsmoking (pack-years) $\mathrm{P}=.001 ; 1$ (rs12045862 x physical activity) $\mathrm{P}=.001 ; 3$ (rs709157 $\mathrm{x}$ time spent sitting) $\mathrm{P}=.001 ; 3$ (rs1175540 x time spent sitting) $\mathrm{P}=.001$; MTMR9; NPCI (rs1805081); MAF (rs1424233); GPRC5B (proximity variant); MC4R (susceptibility loci); POMC; SH2B1; BDNF; FTO; IRS1. New loci/decreasing body fat percentage; decreased IRSI expression, impaired metabolic profile, increased visceral to subcutaneous fat ratio, insulin resistance, dyslipidemia, diabetes risk, and coronary artery may be discovered (Edwards, 2012; Feitosa et al., 2002; Hsueh et al., 2001; Kilpeläinen et al., 2011; Loos et al., 2008; Meyre et al., 2009; Qi et al., 2008; Speliotes et al., 2010; Yanagiya et al., 2007).

\section{Phenotype Evaluations}

\section{Subjects}

Signed consent from subjects and approval from our local IRB preceded this study. Up to 142 randomly selected Africans and African American subjects, male and females-ages 18-45 from 4 populations (1-Africans in US 10 yrs or more, 2-Africans in U.S. to yrs or less, 3-African American, 4-African American Gullah); of varying education level (no high school diploma, GED, college degree and graduate degree); socioeconomic status (working class, unemployed, undergraduate and professional students), having parents of West African lineage or both parents African American descent, were recruited from college campuses, community churches, mosques and sporting organizations via newspaper ads, posters, flyers and word of mouth. Subjects filled out a questionnaire on lineage, a survey on food preferences and food frequency.

\section{Phenotypic Measurements}

Body mass, bioimpedence analysis (BIA) and anthropometrical data were collected by means of an examination in the Sports Medicine facility located in the Student Health Centers of two college campuses. Participants wore a hospital gown with underclothing (barefoot, w/underpants, sans the t-shirt for males, women retaining brassieres) A phenotypic profile was established by a trained clinician of six skin-fold, and nine girth and stature measurements. Digital photographs were taken of the participants from the neck down in their underclothing. Somatotype profiles were established (i.e. endo, ecto, mesomorphic) from the data collected. Measurements were evaluated using an ethnic appropriate 4 component model (Swan, Ball, Athena, 2006) of individual tissue composition that determined fat and fat free masses (water, bone mineral density and residual proteins) adjusted for ethnicity.

Bioimpedence Analysis (Aloia et al., 1997, 1998). Mass, fat and FFM was measured by a digital bioimpedence analy- sis scale. Output generated a value (+/-.003) for total body and muscle mass (g), total body water (TBW) and fat (\%).

Girth measurements (9) (Ross et al., 2003; Carter, 2002). Girth measurements $(\mathrm{cm} / \mathrm{mm})$ was taken using steel anthropometric (Rosscraft, White Rock, BC, Canada) tape from the following positions:

Biepycondal humerus (relaxed and flexed), forearm, supine waist (abdominal), umbilicus, erect hip (hip/buttocks), biepycondal femur (thigh), flexed calf, foot width.

Skinfold Thickness (6). Regional body fat masses was evaluated by skinfold thickness using calipers (Harpenden and Lange) in the subscapular, suprasinale, abdominal, tricep, thigh and calf areas.

Stature (Ozaslan et al., 2003). Stature was evaluated by sliding calipers (Campbell $10 \&$ 20/Rosscraft), a headblock and a Segmometer 4 (Rosscraft) in $\mathrm{cm}$ to measure standing, sitting and trochanteric heights, hand length, and lengths of the foot, leg (thigh and lower) and hand.

Somatotyping (1 of 2) (Ross et al., 2003; Carter, 2002). The Heath-Carter method was used to generate a somatotype profile ecto, endo or mesophoric) from 10 anthropometric positions:

1. Body mass (Ross et al., 2003; Carter and Heath, 1990). From a standing position body mass was recorded from a minimally clothed (hospital gown) subject using a Bioimpedence Analysis (BIA) scale (scale and body composition analyzer, Tanita, Arlington Heights, Illinois, USA). Values were estimated to nearest $0.1 \mathrm{~kg}$ and adjusted for clothing.

2. Stretch stature (height) (Ross et al., 2003; Carter 2002). From a standing position height was estimated using a headsquare (Rosscraft) and carpenters retractable tape (Lufkin). Subject was positioned against a wall, maximally erect with their back, heels and gluteals against the surface. The subjects' head was oriented along the Frankfort Plane with the headsquare (Rosscraft) resting w/ gentle pressure against the hair onto the vertex. Measurements were recorded to the nearest (mm).

3. Tricep Skinfold (Ross et al., 2003; Carter 2002). From a standing position and arms at sides, triceps skinfold was taken from a raised vertical section of the back of the tricep between the acromion and olecranion using a skinfold caliper (Harpenden). Values were estimated to the nearest $0.1 \mathrm{~mm}$.

4. Subscapular Skinfold (Ross et al., 2003; Carter 2002). From a standing position the subscapular skinfold was taken from the subject 45 degrees from the scapula, $2 \mathrm{~cm}$ diagonal from the scapula using a skinfold caliper (Harpenden). Values were recorded to the nearest 0.1 $\mathrm{mm}$.

5. Supraspinale Skinfold (Ross et al., 2003; Carter 2002). From a standing position supraspinale skinfold was taken from the top of the iliac spine on a medial 45 degree line along the anxillary border. A minimal $(5-7 \mathrm{~cm})$ skinfold amount was evaluated relative to the subject using a skinfold caliper (Harpenden). Values was estimated to the nearest $0.1 \mathrm{~mm}$.

6. Medial Calf Skinfold (Ross et al., 2003; Carter, 2002). 
From a standing position, subject raised the right leg to a 90 degree bent knee position upon a stool. Medial calf skinfold was obtained from the maximal girth site girth site on the medial side of the calf using a skinfold caliper (Harpenden). Values were recorded to the nearest $0.1 \mathrm{~mm}$.

7. Biepicondylar Breadth of Humerus (Ross et al., 2003; Carter, 2002). From a seated position, the subject raised the right humerus and bent it 90 degrees at the elbow. Biepicondylar humerus breadth was recorded from the medial and lateral epicondyles using a sliding bone caliper (Campbell 10). Diameter values were recorded to the nearest $0.5 \mathrm{~mm}$.

8. Biepicondylar Breadth of Femur (Ross et al., 2003; Carter, 2002). From a seated position biepicondylar breadth of the femur was evaluated from the subject. The examiner located the medial and lateral epicondyles from a flexed femur, using a small bone caliper (Campbell 10). The maximum epicondylar distance was taken and the diameter was recorded to the nearest $0.5 \mathrm{~mm}$. values.

9. Flexed Arm Girth (Ross et al., 2003; Carter, 2002). Flexed arm girth was taken along the subject's raised, flexed, right arm, bent to a 90 degree position using a flexible steel tape (Rosscraft). The maximal flexed value was recorded at the highest peak of the tricep. Values were estimated to the nearest $\mathrm{mm}$.

10. Tensed Calf Girth (Ross et al., 2003; Carter, 2002). From a standing position calf girth was taken from the right calf of the subject using a retractable steel tape (Rosscraft). Three to four circumference values was taken along the long axis of the lower leg and the highest circumference value recorded to the nearest $\mathrm{mm}$.

This profile was further utilized to evaluate the tendency of the participants towards mesomorphy using the Heath Carter Somatoype method. Data was plotted on a somatochart and a 2D somatochart was also produced.

Somatotyping (2 of 2). Participants were photographed from the neck down (minimally clothed) with a digital camera $(10 \mathrm{mp})$ against a grid pattern to generate a photoscopic somatogram. This data was supplementary to the general somatoyping to further classify and accommodate the evaluation of a potential mixed proportioned participant.

\section{Adjustments}

Errant assumptions inherent in standardized lean density calculations was adjusted for BF\% by ethnicity using, (Schutte et al., 1984), for Black women, (Wagner and Heyward, 2000) for Black men the following calculations for higher proportions of lean body mass inherent in African/African Americans:

4C Model: (Friedl et al., 1992)

Where: $\mathrm{BF}=$ Body fat; $\mathrm{Db}=$ Body density; $\mathrm{TBW}=$ Total body bone mineral; $\mathrm{BM}$

2C Model: (Schutte et al., 1994)

(Black Women)

Where: lean density $(\mathrm{LD})=1.113 \mathrm{~g} / \mathrm{cm} 3$ Blacks (Schutte et al., 1994) vs.1.100 Whites (Siri 1956)
Table 1. FP Survey Category Tables PCA Analysis of Significant Interactions.

\begin{tabular}{|c|c|}
\hline \multicolumn{2}{|c|}{$\begin{array}{c}\text { FP SURVEY CATEGORY TABLES } \\
\text { PRINCIPAL COMPONENT ANALYSIS } \\
\text { SIGNIFICANT INTERACTIONS }\end{array}$} \\
\hline VARIABLES & $\begin{array}{c}\text { SIGNFICIANCE/ } \\
\text { VARIATION PROPORTION }\end{array}$ \\
\hline \multicolumn{2}{|r|}{ 17. OBESITY } \\
\hline 92owc & $<.0001$ \\
\hline 90owself & .49914 \\
\hline 92owc & $<.0001$ \\
\hline 91nwt & -0.45198 \\
\hline 90owself & $<.0001$ \\
\hline 91nwt & -0.77344 \\
\hline 90owself & $<.0001$ \\
\hline fat_p & .43528 \\
\hline 91 nwt & $<.-0001$ \\
\hline fat & .46351 \\
\hline 91nwt & $<.0001$ \\
\hline Age & -0.43424 \\
\hline fat_p & $<.0001$ \\
\hline BMI & .68101 \\
\hline fat_p & $<.0001$ \\
\hline $89 \mathrm{wt}$ & .64505 \\
\hline
\end{tabular}

Note: Adapted from (Johnson et al., 2019; in press).

Table 2. FP Survey Significant Questions Multiple Regression $(\mathrm{BF} \%)$.

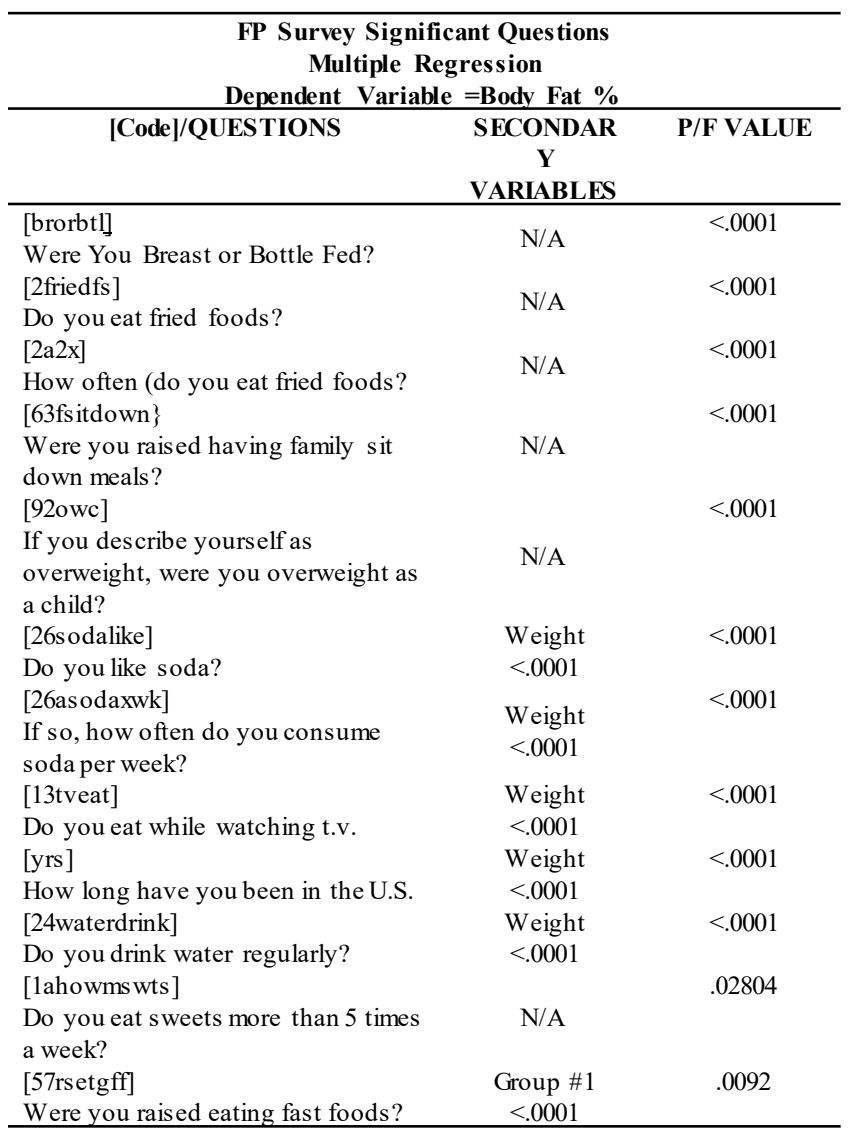

Note: Adapted from (Johnson et al., 2019; in press). 
Table 3. Physiology Data . 4 Component Model Fat Percentages African \& African Americans.

\begin{tabular}{|c|c|c|c|c|c|c|c|c|c|}
\hline \multicolumn{10}{|c|}{$\begin{array}{l}\text { Physiology Data } \\
4 \text { Component Model-Fat Percentages }\end{array}$} \\
\hline ID & Group & $\begin{array}{l}\text { 4C.fat } \\
(\%)\end{array}$ & Age & Gender & ID & Group & $\begin{array}{l}\text { 4C.fat } \\
(\%)\end{array}$ & Age & Gender \\
\hline P13 & Afr $1^{a}$ & 23.4 & 20 & $\mathrm{M}$ & P32 & AA & 24 & 23 & $\mathrm{~F}$ \\
\hline P15 & Afr1 & 11.7 & 26 & M & P34 & AA & 11.6 & 21 & M \\
\hline P18 & Afr1 & 21 & 32 & $\mathrm{~F}$ & P300 & $\mathrm{AA}$ & 30 & 21 & $\mathrm{~F}$ \\
\hline P17 & Afr1 & 34.3 & 38 & M & P313 & AA & 60 & 20 & F \\
\hline P19 & Afr1 & 12.7 & 32 & $\mathrm{~F}$ & P325 & AA & 32 & 23 & $\mathrm{~F}$ \\
\hline P110 & Afr1 & 25.3 & 28 & M & P326 & AA & 29 & 21 & M \\
\hline P111 & Afr1 & 17.9 & 34 & M & P328 & AA & 30 & 25 & M \\
\hline P112 & Afr1 & 11.7 & 26 & M & P329 & AA & 31.2 & 19 & $\mathrm{~F}$ \\
\hline P114 & Afr1 & 24.2 & 36 & M & P332 & AA & 27.7 & 22 & $\mathrm{~F}$ \\
\hline P117 & Afr1 & 17 & 25 & M & P333 & $\mathrm{AA}$ & 25.7 & 25 & $\mathrm{~F}$ \\
\hline P121 & Afr1 & 23 & 27 & M & P334 & AA & 27.2 & 21 & $\mathrm{~F}$ \\
\hline P130 & Afr1 & 31 & 19 & M & P36 & AA & 39 & 33 & $\mathrm{~F}$ \\
\hline P131 & Afr1 & 12.1 & 24 & M & P338 & AA & 25 & 25 & M \\
\hline P135 & Afr1 & 36.9 & 28 & M & P340 & AA & 27.8 & 20 & $\mathrm{~F}$ \\
\hline P136 & Afr1 & 23.8 & 24 & M & P344 & AA & 27 & 20 & M \\
\hline P141 & Afr1 & 16.3 & 21 & M & P345 & AA & 26 & 27 & $\mathrm{~F}$ \\
\hline P144 & Afr1 & 22 & 23 & M & P347 & AA & 26.8 & 20 & M \\
\hline P146 & Afr1 & 11.5 & 21 & M & P349 & $\mathrm{AA}$ & 27.8 & 22 & $\mathrm{~F}$ \\
\hline P148 & Afr1 & 17 & 28 & M & P350 & AA & 26.8 & 25 & M \\
\hline P152 & Afr1 & 22.8 & 29 & M & P351 & $\mathrm{AA}$ & 32.2 & 45 & $\mathrm{~F}$ \\
\hline P154 & Afr1 & 25.1 & 33 & M & P356 & $\mathrm{AA}^{\mathrm{c}}$ & 28 & 22 & M \\
\hline P155 & Afr1 & 19.8 & 29 & M & P361 & $\mathrm{AA}$ & 16.6 & 21 & M \\
\hline P157 & Afr1 & 22.3 & 37 & M & 1P3-12 & AA & 38.9 & 21 & $\mathrm{~F}$ \\
\hline P162 & Afr1 & 18.3 & 24 & M & 2P3A-12 & AA & 25.5 & 20 & $\mathrm{~F}$ \\
\hline P164 & Afr1 & 16.3 & 23 & M & 3P3-12 & AA & 27 & 43 & $\mathrm{~F}$ \\
\hline 1P1-12 & Afr1 & 28.6 & 37 & M & 4P3-12 & AA & 16 & 22 & M \\
\hline $2 \mathrm{P} 1-12$ & Afr1 & 25.3 & 27 & M & $5 \mathrm{P} 3-12$ & AA & 25.4 & 19 & $\mathrm{~F}$ \\
\hline $3 \mathrm{P} 1-12$ & Afrl & 48 & 27 & $\mathrm{~F}$ & $6 \mathrm{P} 3-12$ & AA & 13.6 & 23 & M \\
\hline 1P2-12 & $\mathrm{Afr} 2^{\mathrm{b}}$ & 18.8 & 18 & M & 7P3-12 & AA & 46.4 & 34 & $\mathrm{~F}$ \\
\hline $2 \mathrm{P} 2-12$ & Afr2 & 9.9 & 25 & M & 9P3-12 & AA & 17 & 21 & M \\
\hline $3 \mathrm{P} 2-12$ & Afr2 & 10.6 & 18 & M & 10P3-12 & AA & 31.3 & 19 & $\mathrm{~F}$ \\
\hline $4 \mathrm{P} 2-12$ & Afr2 & 31 & 19 & $\mathrm{~F}$ & 11P3-12 & AA & 41.3 & 29 & M \\
\hline P215 & Afr2 & 16.7 & 21 & $\mathrm{~F}$ & 12P3-12 & $\mathrm{AA}$ & 18 & 20 & $\mathrm{M}$ \\
\hline $\mathrm{P} 237$ & Afr2 & 19.8 & 20 & $\mathrm{~F}$ & \multirow{7}{*}{\multicolumn{5}{|c|}{$\begin{array}{l}{ }^{\mathrm{a}} \mathrm{Afr} 1=\text { Africans in the U.S. } 10 \text { years or less } \\
{ }^{\mathrm{b}} \mathrm{Afr} 2=\text { Africans in the U.S } 10 \text { years or more } \\
{ }^{\mathrm{c} A A}=\text { Non African Affiliated Black Americans }\end{array}$}} \\
\hline P239 & Afr2 & 17 & 19 & $\mathrm{M}$ & & & & & \\
\hline $\mathrm{P} 242$ & Afr2 & 21 & 28 & $\mathrm{~F}$ & & & & & \\
\hline P243 & Afr2 & 18.8 & 20 & $\mathrm{M}$ & & & & & \\
\hline P253 & Afr2 & 24.2 & 18 & $\mathrm{M}$ & & & & & \\
\hline $\mathrm{P} 260$ & Afr2 & 18 & 33 & $\mathrm{~F}$ & & & & & \\
\hline $\mathrm{P} 263$ & Afr2 & 23 & 19 & $\mathrm{~F}$ & & & & & \\
\hline
\end{tabular}

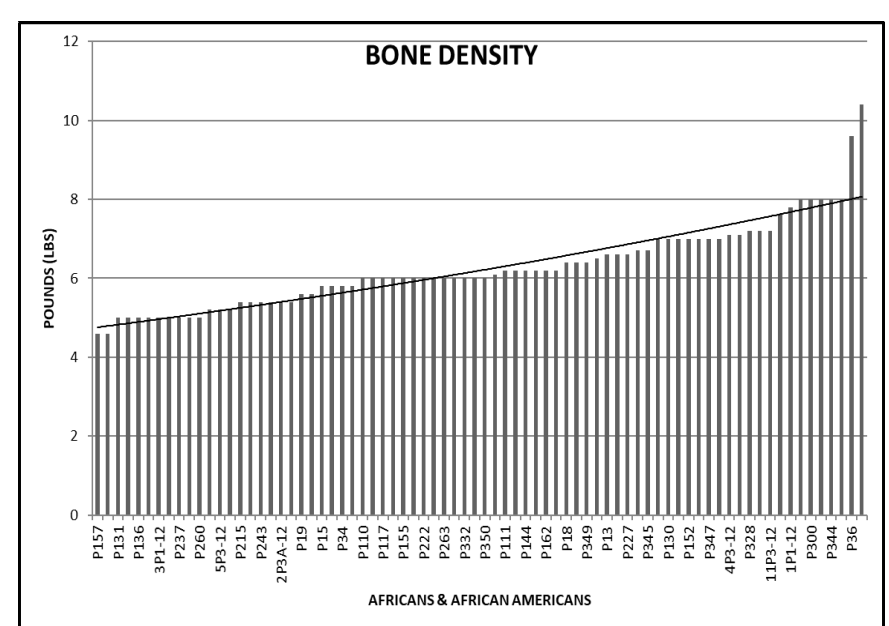

Figure 1. African \& African American Bone Density (Db) in (lbs).

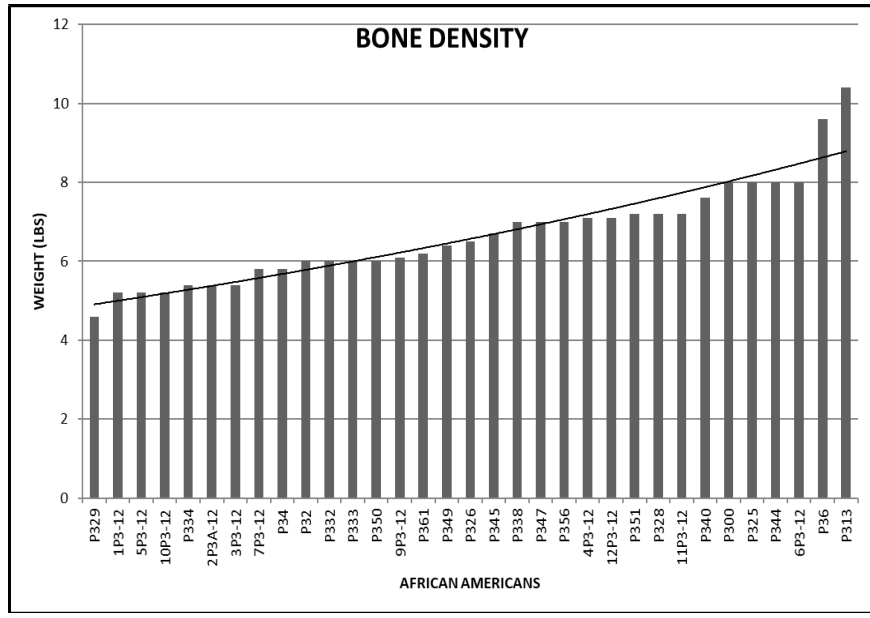

Figure 2. African American Bone Density ( $\mathrm{Db})$ in (lbs). 
Table 4. Four Component Model Physiology Data. Weight in (lbs) for Africans and African Americans.

\begin{tabular}{|c|c|c|c|c|c|c|c|}
\hline \multicolumn{8}{|c|}{$\begin{array}{l}\text { Physiology } \\
\text { 4-Component Model Body Weight (lbs) }\end{array}$} \\
\hline ID & Group & $\begin{array}{l}\text { M.weig } \\
\text { ht } \\
\text { (lbs) }\end{array}$ & Age & ID & Group & $\begin{array}{l}\text { M.weig } \\
\text { ht } \\
\text { (lbs) }\end{array}$ & Age \\
\hline P13 & Afr1 & 173.4 & 20 & P32 & AA & 142 & 23 \\
\hline P15 & Afr1 & 133 & 26 & P34 & AA & 132.8 & 21 \\
\hline P18 & Afr1 & 166 & 32 & P36 & AA & 313.8 & 33 \\
\hline P17 & Afr1 & 214.8 & 38 & P300 & AA & 212 & 21 \\
\hline P19 & Afr1 & 129 & 32 & P313 & AA & 486 & 20 \\
\hline P110 & Afr1 & 163.6 & 28 & P325 & AA & 158 & 23 \\
\hline P111 & Afr1 & 153.4 & 34 & P326 & AA & 200 & 21 \\
\hline P112 & Afr1 & 139.6 & 26 & P328 & AA & 200 & 25 \\
\hline P114 & Afr1 & 174.2 & 36 & P329 & AA & 136.8 & 19 \\
\hline P117 & Afr1 & 161 & 25 & P332 & AA & 190 & 22 \\
\hline P121 & Afr1 & 200 & 27 & P333 & AA & 165.6 & 25 \\
\hline P130 & Afr1 & 219 & 19 & P334 & AA & 150.2 & 21 \\
\hline P131 & Afr1 & 145 & 24 & P338 & AA & 162 & 25 \\
\hline P135 & Afr1 & 164.6 & 28 & P340 & AA & 215 & 20 \\
\hline P136 & Afr1 & 155 & 24 & P344 & AA & 176 & 20 \\
\hline P141 & Afr1 & 147.8 & 21 & P345 & AA & 167 & 27 \\
\hline P144 & Afr1 & 165 & 23 & P347 & AA & 175 & 20 \\
\hline P146 & Afr1 & 121.5 & 21 & P349 & AA & 179.2 & 22 \\
\hline P148 & Afr1 & 220 & 28 & P350 & AA & 170 & 25 \\
\hline P152 & Afr1 & 184 & 29 & P351 & AA & 218.4 & 45 \\
\hline P154 & Afr1 & 169 & 33 & P356 & AA & 165 & 22 \\
\hline P155 & Afr1 & 151 & 29 & P361 & AA & 153.4 & 21 \\
\hline P157 & Afr1 & 120.2 & 37 & 1P3-12 & AA & 170.6 & 21 \\
\hline P162 & Afr1 & 154 & 24 & $2 \mathrm{P} 3 \mathrm{~A}-12$ & AA & 143.2 & 20 \\
\hline P164 & Afr1 & 127 & 23 & $3 P 3-12$ & AA & 157 & 43 \\
\hline 1P1-12 & Afr1 & 223.8 & 37 & 4P3-12 & AA & 137 & 22 \\
\hline $2 \mathrm{P} 1-12$ & Afr1 & 165 & 27 & $5 P 3-12$ & AA & 137.8 & 19 \\
\hline $3 P 1-12$ & Afr1 & 188 & 27 & $6 \mathrm{P} 3-12$ & AA & 188.6 & 23 \\
\hline $1 P 2-12$ & Afr2 & 120.8 & 18 & 7P3-12 & $\mathrm{AA}$ & 213.4 & 34 \\
\hline $2 \mathrm{P} 2-12$ & Afr2 & 180.8 & 25 & $9 P 3-12$ & AA & 145 & 21 \\
\hline $3 P 2-12$ & Afr2 & 147.8 & 18 & $10 \mathrm{P} 3-12$ & AA & 151.4 & 19 \\
\hline $4 P 2-12$ & Afr2 & 160 & 19 & $11 \mathrm{P} 3-12$ & AA & 248.6 & 29 \\
\hline P215 & Afr2 & 132 & 21 & 12P3-12 & AA & 172 & 20 \\
\hline P227 & Afr2 & 219 & 19 & $\mathrm{AFR} 1=$ African & he U.S. 1 & yror less & \\
\hline P237 & Afr2 & 128.6 & 20 & $\mathrm{AFR} 2=$ African & e U.S. 10 & rs or mo & \\
\hline P239 & Afr2 & 130.4 & 19 & $\mathrm{AA}=\mathrm{A}$ frican $\mathrm{A}$ & ans & & \\
\hline P242 & Afr2 & 137 & 28 & & & & \\
\hline P243 & Afr2 & 160 & 20 & & & & \\
\hline P253 & Afr2 & 161.4 & 18 & & & & \\
\hline P260 & Afr2 & 136 & 33 & & & & \\
\hline P263 & Afr2 & 140 & 19 & & & & \\
\hline
\end{tabular}

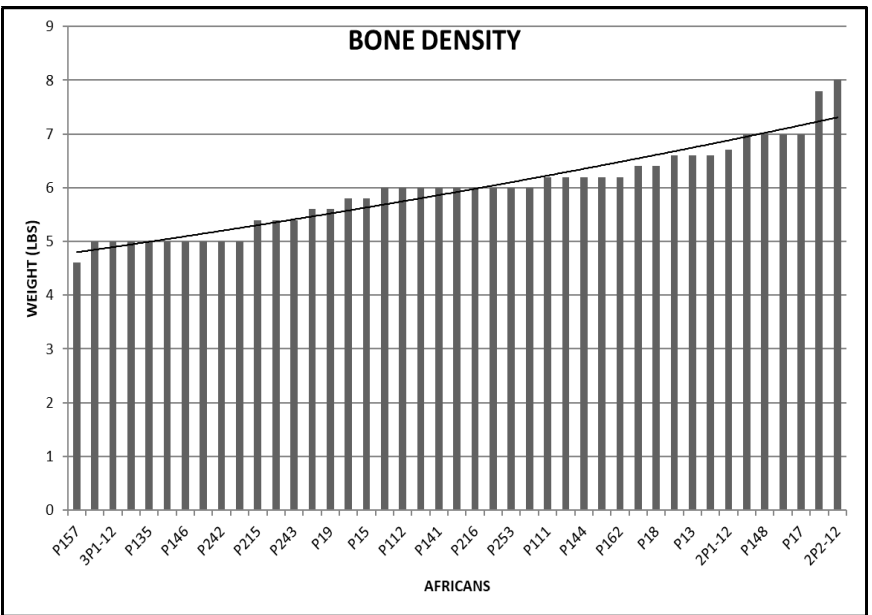

Figure 3. African Bone Density in (lbs).

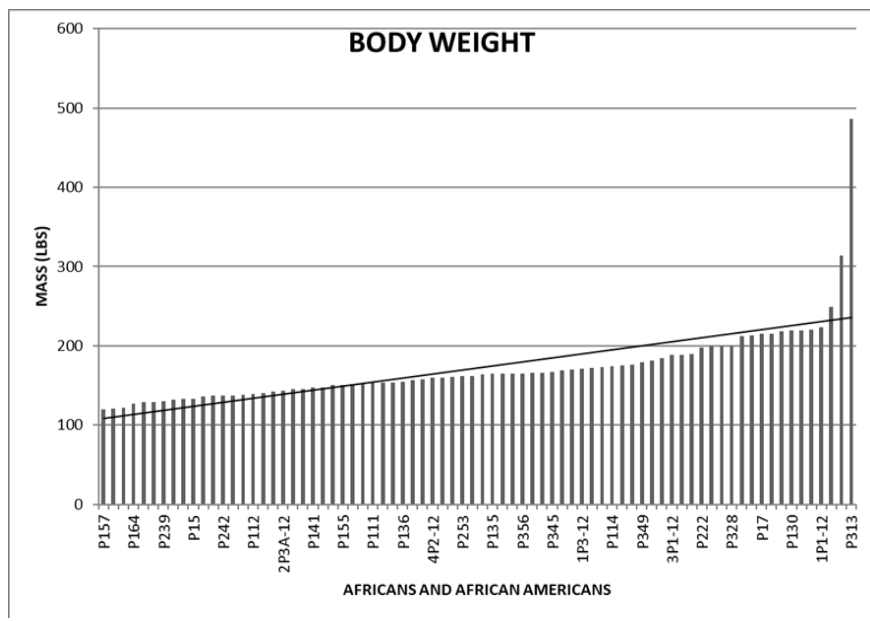

Figure 4. African \& African American Body Weight in (lbs). Data shows physiological agreement of African Americans with reference population of West African. 
Table 5. Physiology Data. 4 Component Model Muscle Weight (lbs) for Africans and African Americans.

\begin{tabular}{|c|c|c|c|c|c|c|c|c|c|}
\hline \multicolumn{10}{|c|}{$\begin{array}{l}\text { Physiology } \\
\text { 4-Component Model Muscle Weight (lbs) \& (\%) }\end{array}$} \\
\hline ID & Group & $\begin{array}{c}\text { Muscle } \\
\text { (lbs) }\end{array}$ & $\begin{array}{l}\text { M. Weight } \\
\text { (lbs) }\end{array}$ & $\%$ & ID & Group & $\begin{array}{c}\text { 4C.muscle } \\
\text { (Ibs) }\end{array}$ & $\begin{array}{l}\text { M.weight } \\
\text { (lbs) }\end{array}$ & $\%$ \\
\hline P13 & Afr & 126.2 & 173.4 & 0.727 & P32 & AA & 101.9 & 142 & 0.717 \\
\hline P15 & Afr & 111.6 & 133 & 0.839 & P34 & AA & 112.2 & 132.8 & 0.844 \\
\hline P18 & Afr & 124.7 & 166 & 0.751 & P36 & AA & 181.8 & 313.8 & 0.579 \\
\hline P17 & Afr & 134.1 & 214.8 & 0.624 & P300 & AA & 117 & 212 & 0.55 \\
\hline P19 & Afr & 107.5 & 129 & 0.833 & P313 & $\mathrm{AA}$ & 184 & 486 & 0.378 \\
\hline P110 & Afr & 116.2 & 163.6 & 0.710 & P325 & AA & 99.4 & 158 & 0.629 \\
\hline P111 & Afr & 119.7 & 153.4 & 0.780 & P326 & AA & 135.5 & 200 & 0.677 \\
\hline P112 & Afr & 117.3 & 139.6 & 0.840 & P328 & AA & 132.8 & 200 & 0.664 \\
\hline P114 & Afr & 125.6 & 174.2 & 0.721 & P329 & AA & 89.5 & 136.8 & 0.654 \\
\hline P117 & Afr & 127 & 161 & 0.788 & P332 & AA & 131.4 & 190 & 0.691 \\
\hline P121 & Afr & 147.8 & 200 & 0.739 & P333 & AA & 117 & 165.6 & 0.706 \\
\hline P130 & Afr & 144.1 & 219 & 0.657 & P334 & AA & 103.9 & 150.2 & 0.691 \\
\hline P131 & Afr & 122.5 & 145 & 0.844 & P338 & AA & 114.5 & 162 & 0.706 \\
\hline P135 & Afr & 98.9 & 164.6 & 0.600 & P340 & AA & 147.5 & 215 & 0.686 \\
\hline P136 & Afr & 112.3 & 155 & 0.724 & P344 & AA & 130.5 & 176 & 0.741 \\
\hline P141 & Afr & 117.7 & 147.8 & 0.796 & P345 & AA & 116.9 & 167 & 0.7 \\
\hline P144 & Afr & 122.5 & 165 & 0.742 & P347 & AA & 121.1 & 175 & 0.692 \\
\hline P146 & Afr & 102.5 & 121.5 & 0.843 & P349 & AA & 123 & 179.2 & 0.686 \\
\hline P148 & Afr & 175.6 & 220 & 0.798 & P350 & AA & 118.4 & 170 & 0.696 \\
\hline P152 & Afr & 135 & 184 & 0.733 & P351 & AA & 140.9 & 218.4 & 0.645 \\
\hline P154 & Afr & 120.4 & 169 & 0.712 & P356 & AA & 107 & 165 & 0.648 \\
\hline P155 & Afr & 115.1 & 151 & 0.762 & P361 & AA & 121.7 & 153.4 & 0.793 \\
\hline P157 & Afr & 88.8 & 120.2 & 0.738 & 1P3-12 & AA & 99 & 170.6 & 0.580 \\
\hline P162 & Afr & 119.6 & 154 & 0.776 & 2P3A-12 & AA & 101.2 & 143.2 & 0.706 \\
\hline P164 & Afr & 100.7 & 127 & 0.792 & 3Р3-12 & AA & 109.2 & 157 & 0.695 \\
\hline 1P1-12 & Afr & 152 & 223.8 & 0.679 & 4P3-12 & AA & 108 & 137 & 0.788 \\
\hline 2P1-12 & Afr & 116.6 & 165 & 0.706 & $5 \mathrm{P} 3-12$ & $\mathrm{AA}$ & 97.6 & 137.8 & 0.708 \\
\hline 3P1-12 & Afr & 92.8 & 188 & 0.493 & $6 \mathrm{P} 3-12$ & AA & 155 & 188.6 & 0.821 \\
\hline 1P2-12 & Afr & 93.1 & 120.8 & 0.770 & $7 \mathrm{P} 3-12$ & AA & 109 & 213.4 & 0.510 \\
\hline 2P2-12 & Afr & 154.9 & 180.8 & 0.856 & 9P3-12 & AA & 114.2 & 145 & 0.787 \\
\hline 3P2-12 & Afr & 125.5 & 147.8 & 0.849 & 10P3-12 & AA & 98.8 & 151.4 & 0.652 \\
\hline 4P2-12 & Afr & 104.6 & 160 & 0.653 & $11 \mathrm{P} 3-12$ & $\mathrm{AA}$ & 138.8 & 248.6 & 0.558 \\
\hline P215 & Afr & 104.7 & 132 & 0.793 & $12 \mathrm{P} 3-12$ & AA & 133.9 & 172 & 0.778 \\
\hline P216 & Afr & 105 & 152 & 0.690 & & & & & \\
\hline P222 & Afr & 118.7 & 198 & 0.599 & & & & & \\
\hline P227 & Afr & 124.8 & 219 & 0.569 & & & & & \\
\hline P237 & Afr & 98.1 & 128.6 & 0.762 & & & & & \\
\hline P239 & Afr & 102.8 & 130.4 & 0.788 & & & & & \\
\hline P242 & Afr & 103.2 & 137 & 0.753 & & & & & \\
\hline P243 & Afr & 124.5 & 160 & 0.778 & & & & & \\
\hline P253 & Afr & 116.3 & 161.4 & 0.720 & & & & & \\
\hline P260 & Afr & 106.5 & 136 & 0.783 & & & & & \\
\hline P263 & Afr & 101.8 & 140 & 0.727 & & & & & \\
\hline
\end{tabular}

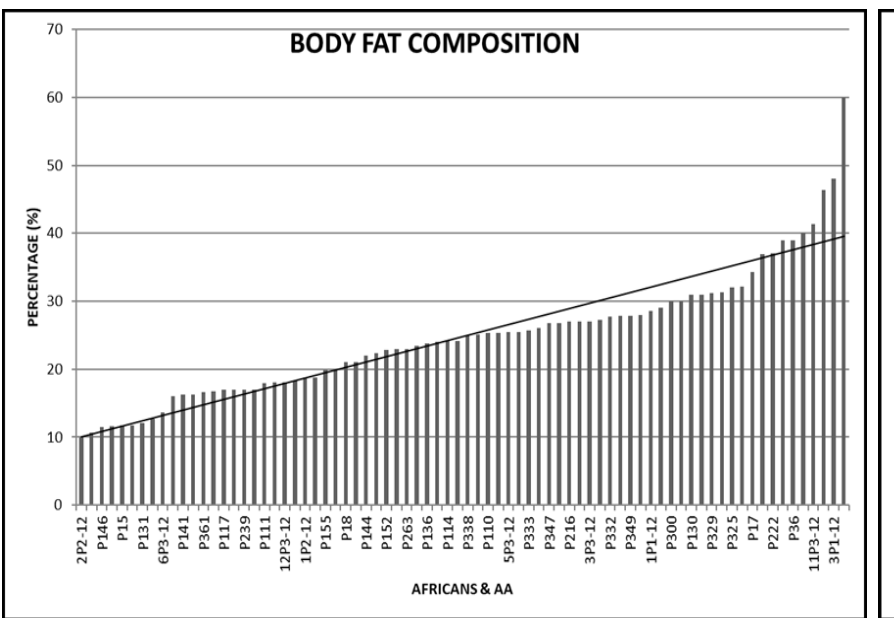

Figure 5. African \& African American Body Fat Composition (\%). Data shows African American agreement with West African reference population. Body fat comprises less than 30 percent of total body mass.

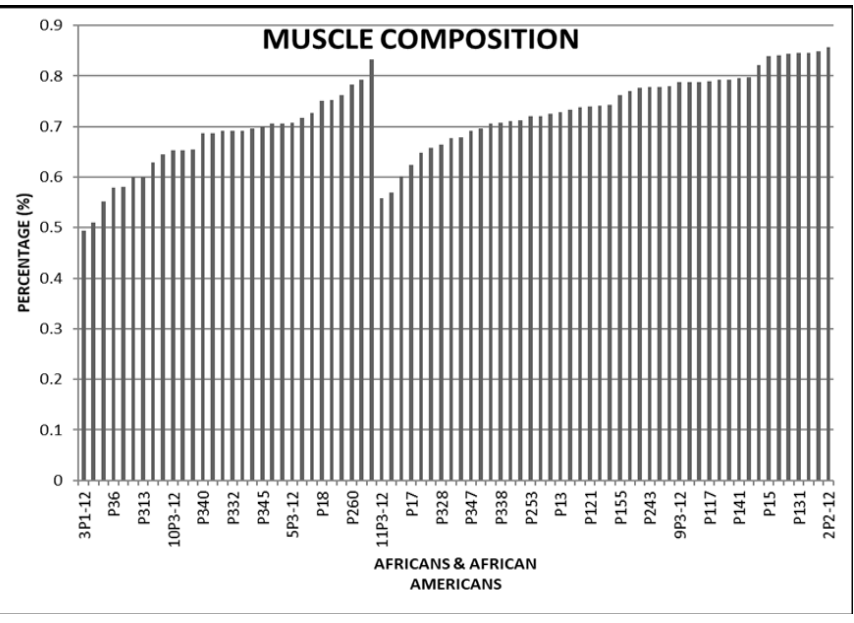

Figure 6. African \& African American Muscle Compostion (\%). Both groups showed agreement with mesomorphic phenotype w/ muscle composition in excess of $60 \%$ of total body mass. 
Table 6. Physiology Data. Group BMI (\%) for Africans and African Americans.

\begin{tabular}{|c|c|c|c|c|c|c|c|c|c|}
\hline \multicolumn{10}{|c|}{$\begin{array}{l}\text { Physiology Data } \\
\text { African and African American Group BMI (\%) }\end{array}$} \\
\hline ID & Group & BMI & $\begin{array}{c}\text { Gende } \\
\mathbf{r}\end{array}$ & Age & ID & Group & BMI & $\begin{array}{c}\text { Gende } \\
\mathbf{r}\end{array}$ & Age \\
\hline $\mathrm{P} 13$ & Afr1 & 21.9 & $\mathrm{M}$ & 20 & P32 & $\mathrm{AA}$ & 22.1 & $\mathrm{~F}$ & 23 \\
\hline $\mathrm{P} 15$ & Afrl & 19.7 & M & 26 & P34 & AA & 18.9 & M & 21 \\
\hline $\mathrm{P} 18$ & Afrl & 32 & $\mathrm{~F}$ & 32 & P36 & AA & 62.8 & $\mathrm{~F}$ & 33 \\
\hline P17 & Afr1 & 29.4 & M & 38 & P300 & AA & 27 & $\mathrm{~F}$ & 21 \\
\hline P19 & Afr1 & 24 & $\mathrm{~F}$ & 32 & P313 & AA & 75.4 & $\mathrm{~F}$ & 20 \\
\hline $\mathrm{P} 110$ & Afrl & 22.6 & $M$ & 28 & P325 & AA & 28.6 & $\mathrm{~F}$ & 23 \\
\hline $\mathrm{P} 111$ & Afrl & 22.6 & M & 34 & P326 & $\mathrm{AA}$ & 28 & $\mathrm{M}$ & 21 \\
\hline P112 & Afr1 & 23 & $M$ & 26 & P328 & AA & 37.4 & $M$ & 25 \\
\hline P114 & Afrl & 25.7 & $M$ & 36 & P329 & $\mathrm{AA}$ & 21 & $\mathrm{~F}$ & 19 \\
\hline P117 & Afr1 & 21 & M & 25 & P332 & AA & 32.4 & F & 22 \\
\hline P121 & Afr1 & 27.1 & $\mathrm{M}$ & 27 & P333 & AA & 27.3 & $\mathrm{~F}$ & 25 \\
\hline $\mathrm{P} 130$ & Afrl & 31.2 & M & 19 & P334 & AA & 24.7 & $\mathrm{~F}$ & 21 \\
\hline $\mathrm{P} 131$ & Afrl & 21.8 & $\mathrm{M}$ & 24 & P338 & $\mathrm{AA}$ & 22 & $\mathrm{M}$ & 25 \\
\hline $\mathrm{P} 135$ & Afrl & 26.9 & $\mathrm{M}$ & 28 & P340 & AA & 32.8 & $\mathrm{~F}$ & 20 \\
\hline $\mathrm{P} 136$ & Afr1 & 42.9 & $M$ & 24 & P344 & AA & 27 & $\mathrm{M}$ & 20 \\
\hline P141 & Afrl & 23.2 & $M$ & 21 & P345 & AA & 27 & $\mathrm{~F}$ & 27 \\
\hline P144 & Afr1 & 24.8 & $\mathrm{M}$ & 23 & P347 & AA & 23 & $\mathrm{M}$ & 20 \\
\hline P146 & Afrl & 21.1 & M & 21 & P349 & AA & 27.2 & $\mathrm{~F}$ & 22 \\
\hline P148 & Afr1 & 29.9 & M & 28 & P350 & $\mathrm{AA}$ & 31.9 & $\mathrm{M}$ & 25 \\
\hline P152 & Afr1 & 26.5 & M & 29 & P351 & $\mathrm{AA}$ & 42 & $\mathrm{~F}$ & 45 \\
\hline $\mathrm{P} 154$ & Afr1 & 25.6 & $M$ & 33 & P356 & AA & 28 & $\mathrm{M}$ & 22 \\
\hline $\mathrm{P} 155$ & Afr1 & 25.2 & $M$ & 29 & P361 & AA & 23.4 & $M$ & 21 \\
\hline P157 & Afrl & 22.3 & $\mathrm{M}$ & 37 & 1P3-12 & AA & 27.9 & $\mathrm{~F}$ & 21 \\
\hline P162 & Afr1 & 24.2 & $\mathrm{M}$ & 24 & 2P3A-12 & AA & 23 & $\mathrm{~F}$ & 20 \\
\hline P164 & Afrl & 20.9 & $\mathrm{M}$ & 23 & 3P3-12 & AA & 26.3 & $\mathrm{~F}$ & 43 \\
\hline 1P1-12 & Afr1 & 32.2 & $\mathrm{M}$ & 37 & 4P3-12 & $\mathrm{AA}$ & 20.6 & $M$ & 22 \\
\hline $2 \mathrm{P} 1-12$ & Afr1 & 30.9 & $M$ & 27 & $5 \mathrm{P} 3-12$ & $\mathrm{AA}$ & 20.4 & $\mathrm{~F}$ & 19 \\
\hline $3 \mathrm{P} 1-12$ & Afr1 & 30 & $\mathrm{~F}$ & 27 & $6 \mathrm{P} 3-12$ & $\mathrm{AA}$ & 26 & $\mathrm{M}$ & 23 \\
\hline 1P2-12 & Afr2 & 20.5 & M & 18 & 7P3-12 & $\mathrm{AA}$ & 39 & $\mathrm{~F}$ & 34 \\
\hline $2 \mathrm{P} 2-12$ & Afr2 & 27.7 & $\mathrm{M}$ & 25 & 9P3-12 & AA & 20.3 & $\mathrm{M}$ & 21 \\
\hline $3 \mathrm{P} 2-12$ & Afr2 & 21.4 & $\mathrm{M}$ & 18 & 10P3-12 & AA & 23.3 & $\mathrm{~F}$ & 19 \\
\hline $4 \mathrm{P} 2-12$ & Afr2 & 28.9 & $\mathrm{~F}$ & 19 & 11P3-12 & $\mathrm{AA}$ & 35.6 & M & 29 \\
\hline P215 & Afr2 & 20.4 & $\mathrm{~F}$ & 21 & $12 \mathrm{P} 3-12$ & $\mathrm{AA}$ & 22.6 & $\mathrm{M}$ & 20 \\
\hline $\mathrm{P} 216$ & Afr2 & 16.5 & $\mathrm{~F}$ & 18 & & & & & \\
\hline P227 & Afr2 & 34.4 & $\mathrm{M}$ & 19 & & & & & \\
\hline P237 & Afr2 & 22.2 & $\mathrm{~F}$ & 20 & & & & & \\
\hline P239 & Afr2 & 19.1 & $\mathrm{M}$ & 19 & & & & & \\
\hline P242 & Afr2 & 20.6 & $\mathrm{~F}$ & 28 & & & & & \\
\hline P243 & Afr2 & 30 & $\mathrm{M}$ & 20 & & & & & \\
\hline P253 & Afr2 & 25.3 & $M$ & 18 & & & & & \\
\hline P260 & Afr2 & 21.2 & $\mathrm{~F}$ & 33 & & & & & \\
\hline P263 & Afr2 & 22.6 & $\mathrm{~F}$ & 19 & & & & & \\
\hline
\end{tabular}

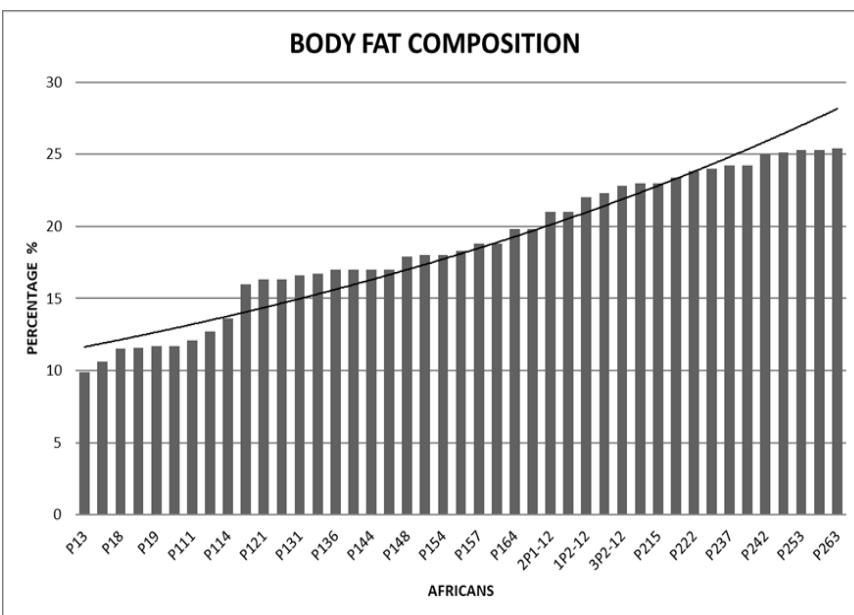

Figure 7. African Body Fat Percentages. Fat mass accounted for less than 30 percent of total body mass in West Africans.

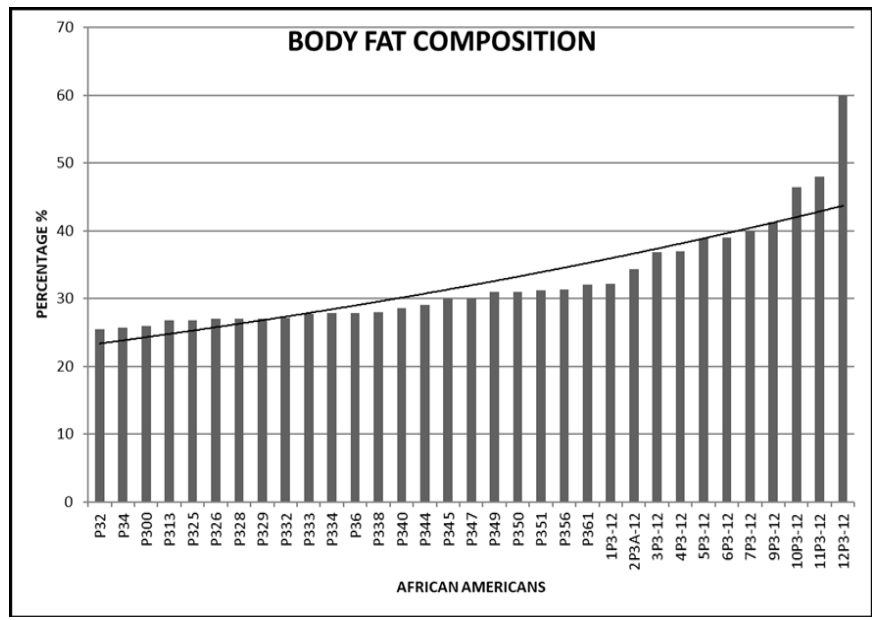

Figure 8. African American Body Fat Percentage. Body fat accounted for 30-40 percent of actual body mass in African Americans. 
Table 7. Significant Variables for Physiology Data.

\begin{tabular}{|c|c|c|c|c|}
\hline \multicolumn{4}{|c|}{$\begin{array}{l}\text { Table of Significant Variables } \\
\text { For Physiology Data }\end{array}$} & \multirow{2}{*}{$\begin{array}{l}\text { One Way Anova (OWA) } \\
\text { =AFR X AA } \\
\text { Linear Regression (LR) } \\
=\text { Var1 x Var } 2 \\
\text { Multiple Regression (MR) } \\
=\text { Dependent Var x V1, V2, V3, V4, V5.... } \\
\text { Data }\end{array}$} \\
\hline Variable & $\begin{array}{l}\text { Dependent } \\
\text { Variable }\end{array}$ & $\mathrm{P} / \mathrm{F}$ Value & Significance & \\
\hline BMI & Muscle lbs & $<.0001$ & Highly Significant & Multiple Regression \\
\hline BMI & Muscle $\%$ & $<.0001$ & Highly Significant & Multiple Regression \\
\hline BMI & & $<.0001$ & Highly Significant & Multiple Regression \\
\hline BMI & Bones & $<.0001$ & Highly Significant & Multiple Regression \\
\hline BMIXFAT & & $<.0001$ & Highly Significant & Linear Regression \\
\hline Bone Density X BMI & & $<.0001$ & Highly Significant & Linear Regression \\
\hline Bones & Muscle lbs & $<.0001$ & Highly Significant & Multiple Regression \\
\hline Bones & BMI & $<.0001$ & Highly Significant & Multiple Regression \\
\hline Bones & Intercept & $<.0001$ & Highly Significant & Multiple Regression \\
\hline Fat & Muscle $\%$ & $<.0001$ & Highly Significant & Multiple Regression \\
\hline Muscle $\%$ & Intercept & $<.0001$ & Highly Significant & Multiple Regression \\
\hline Muscle lbs & & $<.0001$ & Highly Significant & Multiple Regression \\
\hline Weight & BMI & $<.0001$ & Highly Significant & Multiple Regression \\
\hline Fat & & 0.0002 & Very Significant & Multiple Regression \\
\hline Fat & & 0.0003 & Very Significant & Multiple Regression \\
\hline Fat & & 0.0006 & Very Significant & Multiple Regression \\
\hline Group 1 (Afr) & Fat & 0.0002 & Very Significant & Multiple Regression \\
\hline Muscle lbs & Intercept & 0.0027 & Significant & Multiple Regression \\
\hline Body Fat $\%$ & & 0.0039 & Significant & One Way Anova \\
\hline Fat Composition & BMI & 0.006 & Significant & Multiple Regression \\
\hline Group 2 & Bones & 0.0061 & Significant & Multiple Regression \\
\hline Fat & Muscle lbs & 0.0071 & Significant & Multiple Regression \\
\hline BMI & Intercept & 0.0072 & Significant & Multiple Regression \\
\hline BMI & Intercept & 0.009 & Significant & Multiple Regression \\
\hline BMI & Fat & 0.0097 & Significant & Multiple Regression \\
\hline Fat & BMI & 0.01 & Significant & Multiple Regression \\
\hline Weight & & 0.0246 & Significant & One Way Anova \\
\hline Gender & Fat & 0.0366 & Significant & Multiple Regression \\
\hline Group 1 (Afr) & Bones & 0.0367 & Significant & Multiple Regression \\
\hline $\begin{array}{l}\text { Gender } \\
\text { BMI }\end{array}$ & Fat & $\begin{array}{l}0.0372 \\
0.0481\end{array}$ & $\begin{array}{l}\text { Significant } \\
\text { Significant }\end{array}$ & $\begin{array}{l}\text { Multiple Regression } \\
\text { One Way Anova }\end{array}$ \\
\hline
\end{tabular}

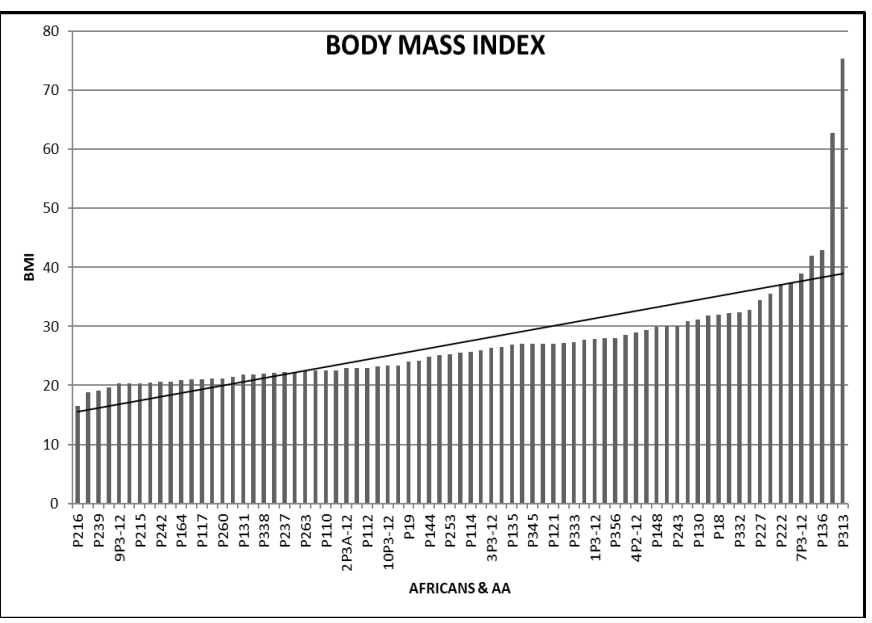

Figure 9. African and African Americans BMI (\%).

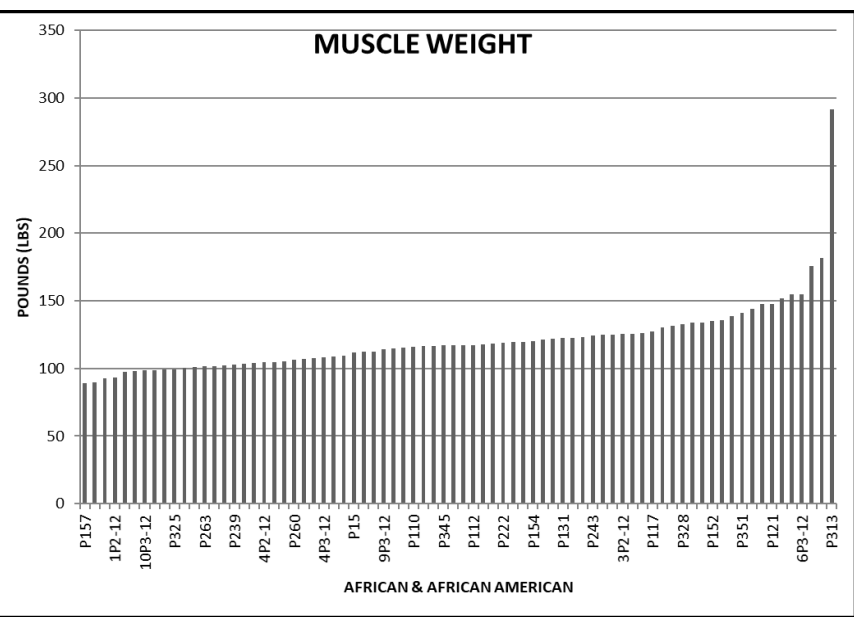

Figure 10. African \& African American Muscle Weights (lbs). 
Table 8. Physiology Data . 4 Component Model Fat Percentages African \& African Americans.

\begin{tabular}{|c|c|c|c|c|c|c|c|c|c|}
\hline \multicolumn{10}{|c|}{$\begin{array}{l}\text { Physiology Data } \\
4 \text { Component Model-Fat Percentages }\end{array}$} \\
\hline ID & Group & $\begin{array}{l}\text { 4C.fat } \\
(\%)\end{array}$ & Age & Gender & ID & Group & $\begin{array}{c}\text { 4C.fat } \\
(\%)\end{array}$ & Age & Gender \\
\hline P13 & $\mathrm{Afr}^{\mathrm{a}}$ & 23.4 & 20 & $\mathrm{M}$ & P32 & $\mathrm{AA}$ & 24 & 23 & $\mathrm{~F}$ \\
\hline P15 & Afr1 & 11.7 & 26 & $\mathrm{M}$ & P34 & $\mathrm{AA}$ & 11.6 & 21 & $\mathrm{M}$ \\
\hline P18 & Afr1 & 21 & 32 & $\mathrm{~F}$ & P300 & $\mathrm{AA}$ & 30 & 21 & $\mathrm{~F}$ \\
\hline P17 & Afr1 & 34.3 & 38 & $\mathrm{M}$ & P313 & $\mathrm{AA}$ & 60 & 20 & $\mathrm{~F}$ \\
\hline P19 & Afr1 & 12.7 & 32 & $\mathrm{~F}$ & P325 & $\mathrm{AA}$ & 32 & 23 & $\mathrm{~F}$ \\
\hline P110 & Afr1 & 25.3 & 28 & M & P326 & $\mathrm{AA}$ & 29 & 21 & M \\
\hline P111 & Afr1 & 17.9 & 34 & $\mathrm{M}$ & P328 & $\mathrm{AA}$ & 30 & 25 & $\mathrm{M}$ \\
\hline P112 & Afr1 & 11.7 & 26 & $\mathrm{M}$ & P329 & $\mathrm{AA}$ & 31.2 & 19 & $\mathrm{~F}$ \\
\hline P114 & Afr1 & 24.2 & 36 & M & P332 & $\mathrm{AA}$ & 27.7 & 22 & $\mathrm{~F}$ \\
\hline P117 & Afr1 & 17 & 25 & $\mathrm{M}$ & P333 & $\mathrm{AA}$ & 25.7 & 25 & $\mathrm{~F}$ \\
\hline P121 & Afr1 & 23 & 27 & M & P334 & $\mathrm{AA}$ & 27.2 & 21 & $\mathrm{~F}$ \\
\hline P130 & Afrl & 31 & 19 & $\mathrm{M}$ & P36 & $\mathrm{AA}$ & 39 & 33 & $\mathrm{~F}$ \\
\hline P131 & Afr1 & 12.1 & 24 & $\mathrm{M}$ & P338 & $\mathrm{AA}$ & 25 & 25 & $\mathrm{M}$ \\
\hline P135 & Afr1 & 36.9 & 28 & M & P340 & $\mathrm{AA}$ & 27.8 & 20 & $\mathrm{~F}$ \\
\hline P136 & Afr1 & 23.8 & 24 & $\mathrm{M}$ & P344 & $\mathrm{AA}$ & 27 & 20 & $\mathrm{M}$ \\
\hline P141 & Afr1 & 16.3 & 21 & $\mathrm{M}$ & P345 & $\mathrm{AA}$ & 26 & 27 & $\mathrm{~F}$ \\
\hline P144 & Afr1 & 22 & 23 & $\mathrm{M}$ & P347 & $\mathrm{AA}$ & 26.8 & 20 & $\mathrm{M}$ \\
\hline P146 & Afr1 & 11.5 & 21 & $\mathrm{M}$ & P349 & $\mathrm{AA}$ & 27.8 & 22 & $\mathrm{~F}$ \\
\hline P148 & Afr1 & 17 & 28 & $\mathrm{M}$ & P350 & $\mathrm{AA}$ & 26.8 & 25 & $\mathrm{M}$ \\
\hline P152 & Afr1 & 22.8 & 29 & $\mathrm{M}$ & P351 & $\mathrm{AA}$ & 32.2 & 45 & $\mathrm{~F}$ \\
\hline P154 & Afr1 & 25.1 & 33 & M & P356 & $\mathrm{AA}^{\mathrm{c}}$ & 28 & 22 & $\mathrm{M}$ \\
\hline P155 & Afr1 & 19.8 & 29 & $\mathrm{M}$ & P361 & $\mathrm{AA}$ & 16.6 & 21 & $\mathrm{M}$ \\
\hline P157 & Afr1 & 22.3 & 37 & $\mathrm{M}$ & 1P3-12 & $\mathrm{AA}$ & 38.9 & 21 & $\mathrm{~F}$ \\
\hline P162 & Afr1 & 18.3 & 24 & $\mathrm{M}$ & $2 \mathrm{P} 3 \mathrm{~A}-12$ & $\mathrm{AA}$ & 25.5 & 20 & $\mathrm{~F}$ \\
\hline P164 & Afr1 & 16.3 & 23 & M & 3Р3-12 & $\mathrm{AA}$ & 27 & 43 & $\mathrm{~F}$ \\
\hline $1 \mathrm{P} 1-12$ & Afr1 & 28.6 & 37 & M & $4 \mathrm{P} 3-12$ & $\mathrm{AA}$ & 16 & 22 & $\mathrm{M}$ \\
\hline 2P1-12 & Afr1 & 25.3 & 27 & M & $5 \mathrm{P} 3-12$ & $\mathrm{AA}$ & 25.4 & 19 & $\mathrm{~F}$ \\
\hline $3 \mathrm{P} 1-12$ & Afr1 & 48 & 27 & $\mathrm{~F}$ & $6 \mathrm{P} 3-12$ & $\mathrm{AA}$ & 13.6 & 23 & M \\
\hline 1P2-12 & $\mathrm{Afr} 2^{\mathrm{b}}$ & 18.8 & 18 & M & 7P3-12 & $\mathrm{AA}$ & 46.4 & 34 & $\mathrm{~F}$ \\
\hline 2P2-12 & Afr2 & 9.9 & 25 & M & 9P3-12 & $\mathrm{AA}$ & 17 & 21 & M \\
\hline $3 \mathrm{P} 2-12$ & Afr2 & 10.6 & 18 & M & 10P3-12 & $\mathrm{AA}$ & 31.3 & 19 & $\mathrm{~F}$ \\
\hline $4 \mathrm{P} 2-12$ & Afr2 & 31 & 19 & $\mathrm{~F}$ & $11 \mathrm{P} 3-12$ & $\mathrm{AA}$ & 41.3 & 29 & M \\
\hline $\mathrm{P} 215$ & Afr2 & 16.7 & 21 & $\mathrm{~F}$ & 12P3-12 & $\mathrm{AA}$ & 18 & 20 & $\mathrm{M}$ \\
\hline P237 & Afr2 & 19.8 & 20 & $\mathrm{~F}$ & \multirow{7}{*}{\multicolumn{5}{|c|}{ 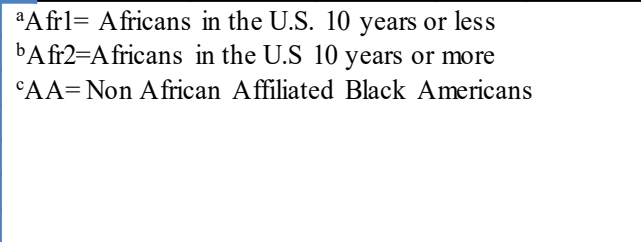 }} \\
\hline P239 & Afr2 & 17 & 19 & $\mathrm{M}$ & & & & & \\
\hline P242 & Afr2 & 21 & 28 & $\mathrm{~F}$ & & & & & \\
\hline P243 & Afr2 & 18.8 & 20 & $\mathrm{M}$ & & & & & \\
\hline $\mathrm{P} 253$ & Afr2 & 24.2 & 18 & $\mathrm{M}$ & & & & & \\
\hline P260 & Afr2 & 18 & 33 & $\mathrm{~F}$ & & & & & \\
\hline P263 & Afr2 & 23 & 19 & $\mathrm{~F}$ & & & & & \\
\hline
\end{tabular}

$2 C$ Model: Wagner et al. $\{\% \mathrm{BF}=[(4.858 / \mathrm{Db})-4.394] \times 100$ (Wagner and Heyward, 2000)

(Black Men)

Additionally, data was adjusted for age and gender.

\section{Statistical Analysis}

Phenotypic data was evaluated among the three groups where:

Group 1: Reference population of African Americans-West Africans in US less than $10 \mathrm{yrs}$

Group Two: Reference population for African Americans- West African in the US 10 years or more

Group Three: African Americans

Statistical analysis was used to determine the significance of the variances among the groups. Phenotypic data (mass, heights, breadths, girths, skinfold thicknesses) were used as variables
(32). Statistical significance was obtained among variables using ANOVA (SAS Inc. Cary, NC). Significant variables shown on Table 1 were ranked using Principal Component Analysis (SAS) and Table 2 shows further analysis on a regression curve using Multiple Regression (SAS).

\section{Self-Reported Physiology Survey Response Data}

\section{Non/Normal Weight (Self- Reported)}

The majority of Africans and African Americans described themselves as normal weight. A larger percentage of Africans described themselves as normal weight than African Americans. About $61 \%$ of African Americans described themselves as normal weight, $39 \%$ did not (Table 2). Almost $79 \%$ of Africans described themselves as normal weight, $21 \%$ did not. 


\section{Overweight (Self-reported)}

Among African Americans, $61 \%$ did not describe themselves as overweight, $39 \%$ did. About $81 \%$ of Africans did not describe themselves as overweight, $16 \%$ did (Table 1).

\section{Childhood Overweight (Self-reported)}

The majority of African and African Americans were not overweight as children. More African Americans were overweight as children than Africans. About $64 \%$ of African Americans said they were not overweight as children, $21 \%$ couldn't remember (Table 2). Just 18\% of African Americans said they were overweight as children. Among African groups, $60 \%$ said they were not overweight as children, $32 \%$ didn't remember. Just $5 \%$ of Africans said they were overweight as children (Table 2). Therefore, there may be a population at risk for adult obesity due to childhood obesity.

\section{Parents}

Participants were asked if either of their parents were overweight. About $59 \%$ of all groups responded that their parents were not overweight. Almost $41 \%$ of all groups said their parents were overweight.

The majority of Africans and African Americans said their parents were not overweight. African American parents were reported overweight at the same percentage as they were not reported overweight. More African Americans reported their parents overweight than Africans. Among African Africans, 52\% said their parents were overweight, and the same percentage said their parents were not.

About $65 \%$ of Africans said their parents were not overweight. Just $35 \%$ of African Americans did not have parents who were overweight. Therefore, because a majority population of African Americans reported parents who were overweight, African Americans are at risk of overweight due to parental inheritance of BMI (Danielzik et al., 2002; Robl et al., 2008).

\section{Prevention/Breast Feeding}

Almost all Africans were breast fed and not even half of African Americans were. Among African Americans 42\% were not breast fed and $45 \%$ were. Just $12 \%$ of African Americans couldn't remember. About $95 \%$ of Africans were breast fed, $5 \%$ were not (Table 2). Therefore, African Americans are were more at risk of childhood and adult obesity due to lack of breastfeeding than Africans (Dewey, 2003).

\section{Bone Density}

There was variation in bone density $(\mathrm{Db})$ between African participants in the U.S. ten years or more (tenplus) and those who had been in the U.S. 10 years or less (tenless). The former had lighter skeletal weights or bone density and the latter had heavier bone density. African Americans had the heaviest bone density (Table 3). It seems to suggest a relationship between time in the U.S. and bone density in Africans and African
Americans (Figures 1-3; Suppl. Figures A1 and A2). Perhaps there are characteristics of foods grown in American soils that is related to this phenomenon. A future study might examine the relationship between U.S. soil nutrients and its effect on bone mineral density of immigrant populations like Africans, over time.

\section{African American and African Weights vs. Gender}

African Americans females weighed more on average than African American males and Africans. African males weighed more than African females (Tables 6-7). More than $60 \%$ of their body mass was attributed to muscle (Figures 4-6; Suppl. Figuress A3-6, A7-14).

\section{Fat Percentage}

About $72 \%$ of African Americans were found to be over-fat by the standard of hydrodensitometry (Smalley et al) and 58\% of Africans. However, when adjusted for cultural acceptance, based on the participant's response of themselves as overweight ( $39 \%$ of AAs; $16 \%$ of Afr), the total was corrected by respondents whose body fat exceeded $31 \%$. The total number of overfat went from $72 \%$ African Americans to $9 \%$ and $58 \%$ Africans to $2 \%$ (Figures 1-3; Table 7).

\section{Conclusion}

This study asked the question: "if BMI is a proper assessment tool to measure obesity among African and African American populations" and it is not. According to the BMI standard $48 \%$ of both of these groups would be classified as overweight and obese with African Americans comprising $41 \%$ of this category and Africans comprising even more at $45 \%$ (Table 8 , Figures $5,7-8)$.

1. BMI falsely assumed the presence of fat. It was assumed that the resultant values for mass in this population correlated with fat. It did not (Tables 6-9).

2. A breakdown of values into a 4-component model (body fat percentage, body water, bone density and muscle mass) was more informative towards adiposity (Tables 3-8, Suppl. Tables A1-A2; Figures 1-10; Suppl. Figures A1-34).

3. Body Fat Percentage among the groups was at $24.7 \%$ overall and $29.3 \%$ for females, $21.8 \%$ for males, $28.1 \%$ for African Americans and 22.1\% for Africans. According to the BMI tables these values are all within normal range. The hydrodensitometry standard for body fat percentage is $20 \%$ for male and $25 \%$ for female (Smalley et al., 1990). Therefore, $4.3 \%$ of AAs in the study were over-fat and $1.8 \%$ of Africans (Table 7; Figures $5,7-8)$.

4. Fat free mass value of bone density on average comprised 6lbs for Africans and 7lbs for African Americans of total body mass value (Table 3 ).

5. Fat free mass value of Total Body Water (TBW) comprised $37.8 \%$ of body mass value in Africans and Afri- 
can Americans (Suppl. Table A1).

6. Fat-free mass values of Muscle Composition were around $70 \%$ of body mass value for African and African Americans, confirming a mesomorphic somatotype. Muscle comprised 123lbs of weight in African Americans and 118lbs for Africans (Tables 5, 7, and Figure $10)$.

The BMI standard cannot be used accurately to assess adiposity among all cultural groups only within groups. When applied within the comparison of African Americans and their reference population-West Africans, there is stern disagreement in the data that this population is $48 \%$ overweight and obese. The within group comparison showed the BMI value to be within normal range, $\mathrm{w} / 70 \%$ of body mass to be explained by fat free muscle composition. This is in agreement with the cultural standard for Africans and African American somatotype of mesomorphy, having a large component of body mass comprised of muscle mass (Table 6; Suppl. Figures 18-25).

\section{Ideal Weight}

The notion of "ideal weight" is biologically meaningless and represents the efforts of persons well placed politically and well published academically. Height and weight tables are popular, prevalent and standardized but not objective, biologically meaningless, and unscientific.

\section{Statistics}

Multiple Regression analysis (Table 1) using the fat free mass value of muscle weight in lbs as the dependent variable showed it to have a highly significant $(\mathrm{P}=<.0001)$ positive correlation with BMI. Muscle percentage had a highly significant $(\mathrm{P}=<.0001)$ positive correlation with BMI. The fat free mass value of bones, when used a dependent variable in Multiple Regression analysis had a highly significant $(\mathrm{P}=<.0001)$ positive correlation with BMI.

Linear Regression analysis examining Bone Density $(\mathrm{Db})$ as a variable against BMI found it to be a highly significant $(\mathrm{P}=<.0001)$ interaction. Linear Regression analysis examining the interaction between BMI and Fat resulted in a highly significant $(\mathrm{P}=<.0001)$ positive correlation (Table 2$)$.

A Multiple Regression analysis examining the same interaction found it to be a significant positive correlation $(\mathrm{P}=.0060)$ (Kennedy et al., 2009; Smalley et al., 1990).

\section{References}

Ahituv N, N Kavaslar, W Schackwitz, A Ustaszewska, J Martin, S Hébert, H Doelle, B Ersoy, G Kryukov, and S Schmidt (2007) Medical Sequencing at the Extremes of Human Body Mass." The American Journal of Human Genetics 80 (4): 779-791.

Aleman-Mateo H, SY Lee, F Javed, J Thornton, SB Heymsfield, RN Pierson, FX Pi-Sunyer, ZM Wang, J Wang, and D Gallagher (2009) Elderly Mexicans have less muscle \& greater total truncal fat compared to African-Americans \& Caucasians w/ the same BMI. Journal of Nutrition, Health \& Aging 13 (10): 919-923.

Aloia, and et al. (1997) Comparison of body composition in Black and White premenopausal women. Journal of Laboratory and Clinical
Medicine 129 (3): 294-99.

Aloia, J. F., A. Vaswani, E. Flaster, and R. Ma. 1998. "Relationship of body water compartments to age, race, and fat-free mass." J Lab Clin Med no. 132:483-90.

Bagwell, M Allison, JL Bento, JC Mychaleckyj, BI Freedman, CD Langefeld, and DW Bowden (2005) Genetic analysis of HNF4A polymorphisms in Caucasian-American type 2 diabetes. Diabetes 54 (4): 1185-1190.

Barroso I (2005) Genetics of type 2 diabetes. Diabetic Medicine 22 (5): 517-535.

Bjorntorp P (1985) Regional patterns of fat distribution. Annals of Internal Medicine 103 (6 pt 2): 994-995.

Bouchard C, A Tremblay, JP Després, A Nadeau, PJ Lupien, G Thériault, J Dussault, S Moorjani, S Pinault, and G Fournier (1990) The response to long-term overfeeding in identical twins. New England Journal of Medicine 322 (21): 1477-1482.

Branson R, N Potoczna, JG Kral, KU Lentes, MR Hoehe, and FF Horber (2003) Binge eating as a major phenotype of melanocortin 4 receptor gene mutations. New England Journal of Medicine no. 348 (12): 1096-1103.

Calton MA, and C Vaisse (2009) Narrowing down the role of common variants in the genetic predisposition to obesity. Genome medicine 1 (3): 31.

Carroll JF, AL Chiapa, et al. (2008) Visceral fat, waist circumference, and BMI: Impact of race, ethnicity. Obesity 16 (3): 600-607.

Carter JEL, and BH Heath (1990) Somatotyping. Development and Applications. 1st ed, Cambridge Studies in Biological Anthropology. Cambridge: Cambridge University Press.

Chambers JC, P Elliott, D Zabaneh, W Zhang, Y Li, P Froguel, D Balding, J Scott, and JS Kooner (2008) Common genetic variation near MC4R is associated with waist circumference and insulin resistance. Nature Genetics 40 (6): 716.

Daniel M, DS Moore, et al. (2006) Associations among education, cortison rythym, and BMI in blue collar women. Obesity Research 14 (2): $327-335$.

Danielzik S, K Langnase, M Mast, C Spethmann, and MJ Muller (2002) Impact of parental BMI on the manifestation of overweight 5-7 year-old children. Journal of Nutrition 41 (3): 132-138.

Dewey KG (2003) Is breastfeeding protective against child obesity?" Journal of Human Lactation 19 (1): 9-18.

Dong C, WD Li, F Geller, L Lei, D Li, OY Gorlova, J Hebebrand, CI Amos, RD Nicholls, and RA Price (2005) Possible genomic imprinting of three human obesity-related genetic loci. The American Journal of Human Genetics 76 (3): 427-437.

Dong C, S Wang, WD Li, D Li, H Zhao, and RA Price (2003) Interacting genetic loci on chromosomes 20 and 10 influence extreme human obesity. The American Journal of Human Genetics 72 (1): 115-124.

Dubern B, K Clément, V Pelloux, P Froguel, JP Girardet, B Guy-Grand, and P Tounian (2001) Mutational analysis of melanocortin-4 receptor, agouti-related protein, and $\alpha$-melanocyte-stimulating hormone genes in severely obese children. The Journal of pediatrics 139 (2): 204-209.

Duncan AE, A Agrawal, et al. (2009) Genetic and environmental contributions to BMI in adolescent \& young adult women. Obesity 17 (5): 1040-1043.

Durenberg P and Y Durenberg (2001) Differences in body-composition assumptions across ethnic groups: practical consequences. Current Opinions in Clinical Nutrition and Metabolic Care 4: 377-383.

Ebersole KE, LR Dugas, RA Durazo-Arvizu, AA Adeyemo, BO Tayo, OO Omotade, WR Brieger, DA Schoeller, RS Cooper, and AH Luke (2008) Energy expenditure and adiposity in Nigerian and 
African-American women. Obesity 16. doi: 10.1.38/oby.2008.330. Edwards TL, DR Velez Edwards, R Villegas, SS Cohen, MS Buchowski, JH Fowke, D Schlundt, J Long, Q Cai, W Zheng, XO Shu, MK Hargreaves, J Smith, SM Williams, LB Signorello, WJ Blot, and CE Matthews (2012) HTR1B, ADIPOR1, PPARGC1A, and CYP19A1 and obesity in a cohort of Caucasians and African Americans: an evaluation of gene-environment interactions and candidate genes. Am J Epidemid 175 (1): 11-21.

Epel ES, EH Blackburn, J Lin, FS Dhabhar, NE Adler, JD Morrow, and RM Cawthon (2004) Accelerated telomere shortening in response to life stress. Proc Natl Acad Sci USA 101 (49): 17312-5. doi: 10.1073/pnas.0407162101.

Evans EM, DA Rowe, SB Racette, KM Ross, and E McAuley (2006) Is the current BMI obesity classification appropriate for Black and White post-menopausal women? Journal of Obesity 30 (5): 837843.

Farooqi IS, JM Keogh, GSH Yeo, EJ Lank, T Cheetham, and S O'rahilly (2003) Clinical spectrum of obesity and mutations in the melanocortin 4 receptor gene. New England Journal of Medicine 348 (12): 1085-1095.

Feitosa MF, IB Borecki, SS Rich, DK Arnett, P Sholinsky, RH Myers, M Leppert, and MA Province (2002) Quantitative-trait loci influencing body-mass index reside on chromosomes 7 and 13: the National Heart, Lung, and Blood Institute Family Heart Study. The American Journal of Human Genetics 70 (1):7 2-82.

Fox SI (2008) Human physiology. Concepts \& clinical applications. 13th ed. New York: McGraw Hill.

Freedman DS, LK Khan, et al. (2005) The relation of childhood BMI to adult adiposity: The Bogalusa Heart Study. Pediatrics 115 (1 of 2): $22-27$

Freedman DS and B Sherry (2009) The Validity of BMI as an Indicator of Body Fatness and Risk Among Children. Pediatrics 124: S23S34. doi: 10.1542/peds.2008-3586E.

Gallagher CJ, CD Langefeld, CJ Gordon, JK Campbell, JC Mychalecky, M Bryer-Ash, SS Rich, DW Bowden, and MM Sale (2007) Association of the estrogen receptor- $\alpha$ gene with the metabolic syndrome and its component traits in African-American families: the Insulin Resistance Atherosclerosis Family Study. Diabetes 56 (8): 2135-2141.

Gallagher D, M Visser, et al. (1996) How useful is body mass index for comparison of body fat mass across age, sex and ethnic groups. Am J Epidemid 143: 228-239.

Harrison, G. 1985. "Height-weight tables.” Annals of Internal Medicine no. 103 (6 pt 2):989-94.

Hasstedt SJ, ME Ramirez, H Kuida, and RR Williams (1989) Recessive inheritance of a relative fat pattern. American journal of human genetics 45 (6): 917.

Haworth CMA, R Plomin, et al. (2008) Childhood obesity: genetic and environmental overlap w/normal range BMI. Obesity 16 (7): 1585-1590.

Hjelmborg JVB, C Fanani, K Silventoinen, M McGue, M Korkeila, K Christensen, A Rissanen, and M Kaprio (2008) Genetic influences on growth traits of BMI: A longitudinal study of adult twins. Obesity 16 (4): 847-852.

Hortobagyi T, VL Katch, PF LaChance, and AR Behnke (1990) Relationships of body size, segmental dimensions, and ponderal equivalents to muscular strength in high-strength and low-strength subjects. International Journal of Sports Medicine \& Physical Fitness 11 (5): 349-56.

Hsueh, Wen-Chi, Braxton D Mitchell, Jennifer L Schneider, Pamela L St. Jean, Toni I Pollin, Margaret G Ehm, Michael J Wagner, Daniel K Burns, Hakan Sakul, and Callum J Bell. 2001. "Genome-wide scan of obesity in the Old Order Amish." The Journal of Clinical Endocrinology \& Metabolism no. 86 (3):1199-1205.

Hunt, S. C., S. Stove, and et al. 2008. "Association of the FTO gene w/ BMI.” Obesity no. 16 (4):902-904.

Kaarma, H., J. Peterson, J. Kasmel, and et al. 2009. "The role of body height, weight \& BMI in Body build classification." Papers on Anthropology no. 18:155-173.

Kennedy AP, JL Shea, and G Sun (2009) Comparison of the classification of obesity by BMI vs dual-energy x-ray absorptiometry in the New Foundland population. Obesity 17 (11): 2094-2099.

Kilpeläinen TO, MC Zillikens, A Stančákova, FM Finucane, JS Ried, C Langenberg, W Zhang, JS Beckmann, J Luan, and L Vandenput (2011) Genetic variation near IRS1 associates with reduced adiposity and an impaired metabolic profile. Nature Genetics 43 (8): 753.

Kleerokoper M, DA Nelson, et al. (1994) Body composition and gonadal steroids in older White and Black women. Clinical Endocrinol Metab 79: 775-779.

Knapp T (1983) A methodological critique of the 'ideal weight' concept. JAMA 250: 506-510.

Kok P, JC Seidell, and AE Meinders (2004) The value and limitations of the body mass index (BMI) in the assessment of the health risks of overweight and obesity. Nederlands Tijdschrift voor Geneeskunde no. 148 (48): 2379-2382.

Komlos J, A Breitfelder, and M Sunder (2009) The transition to postindustrial BMI values among U.S. children. American Journal of Human Biology 21 (2): 151-160.

Kuczymarksi R and KM Flegal (2000) Criteria for definition of overweight in transition: Background and recommendations for the United States. Am J Clin Nutr 72: 1074-81.

Li L, C Law, R Conte, and C Power (2009) Intergenerational influences on childhood body mass index:The effect of parental body mass index trajectories. Journal of Clinical Nutrition 89 (2): 551-557.

Lin J, E Epel, and E Blackburn (2012) Telomeres and lifestyle factors: Roles in cellular aging. Mutat Res 730 (1-2): 85-9. doi: 10.1016/j. mrfmmm.2011.08.003.

Loos RJF, CM Lindgren, S Li, E Wheeler, JH Zhao, I Prokopenko, M Inouye, RM Freathy, AP Attwood, and JS Beckmann (2008) Common variants near MC4R are associated with fat mass, weight and risk of obesity. Nature Genetics 40 (6): 768.

McAdams MA, RM Dam, and FB Hu (2007) Comparison of self-reported and measured BMI as correlates of disease markers in U.S. adults. Obesity 15 (1): 188-196.

Meyre D, J Delplanque, JC Chèvre, C Lecoeur, S Lobbens, S Gallina, E Durand, V Vatin, F Degraeve, and C Proença (2009) Genomewide association study for early-onset and morbid adult obesity identifies three new risk loci in European populations. Nature Genetics 41 (2): 157

Mills TC, D Gallagher, J Wang, and S Heshka (2007) Modeling the relationship between body fat and the BMI." Int Journal of Body Composition 5 (2): 73-79.

Mott J et al. (1999) Relation between body fat and age in 4 ethnic groups. American Journal of Clinical Nutrition 69: 1007-1013.

Mustelin L, K Silventoinen, et al. (2009) Physical activity reduces the influence of genetic effects on BMI and waist circumference: A study in young adult twins. Int Journal of Obesity 33 (1): 29-36.

Nishigori H, H Tomura, N Tonooka, M Kanamori, S Yamada, K Sho, I Inoue, N Kikuchi, K Onigata, and I Kojima (2001) Mutations in the small heterodimer partner gene are associated with mild obesity in Japanese subjects. Proceedings of the National Academy of Sciences 98 (2): 575-580.

Norman RA, DB Thompson, T Foroud, WT Garvey, PH Bennett, C Bogardus, and E mo Ravussin (1997) Genomewide search for 
genes influencing percent body fat in Pima Indians: suggestive linkage at chromosome 11q21-q22. Pima Diabetes Gene Group. American Journal of Human Genetics 60 (1): 166.

Ohlsson C, N Hellberg, P Parini, O Vidal, M Bohlooly, M Rudling, M Lindberg, M Warner, and JA Gustafsson (2000) Obesity disturbed lipoprotein profile in estrogen receptor (alpha) deficient male mice. Biochemical and Biophysical Research Communications 278: 640645.

Okorodudu DO, MF Jumean, Victor Manuel Montori, A Romero-Corral, VK Somers, PJ Erwin, and F Lopez-Jimenez (2010) Diagnostic performance of body mass index to identify obesity as defined by body adiposity: a systematic review and meta-analysis. International Journal of Obesity 34 (5): 791.

Ortega-Alonso A, S Sipila, et al. (2009) Genetic influences on change in BMI from middle to old ag: A 29-year follow-up study of twin sisters. Behavior Genetics 39 (2): 154-164.

Ozaslan A, MY Iscan, I Ozaslan, H Tugcu, and S Koc (2003) Estimation of stature from body parts. Forensic Science International 3501: 1-6.

Paganini-Hill A, AO Martin, and MA Spence (1981) The S-leut anthropometric traits: Genetic analysis. American Journal of Physical Anthropology 55 (1): 55-67.

Proctor A (2009) Genetics of obesity and diabetes in the IRAS Family Study, Wake Forest University.

Qi L, P Kraft, DJ Hunter, and FB Hu (2008) The common obesity variant near MC4R gene is associated with higher intakes of total energy and dietary fat, weight change and diabetes risk in women. Human Molecular Genetics 17 (22): 3502-3508.

Robl M, I Knerr, KM Keller, R Jaeschke, U Hoffmeister, T Reinehr, and RW Holl (2008) Obesity in children \& adolescents and their parents: correlation of patients' body mass index w/ that of their parents and siblings recorded in the Multicentre APS Study. Deutsche Medizinische Wocherschrift 133 (47): 2448-2453.

Ross WD, RV Carr, JM Guelke, and JEL Carter (2003) Anthropometry Fundamentals: Rosscraft/Turnpike Electronic Publications.

Rothman KJ (2008) BMI related errors in the measurement of obesity. Int Journal of Obesity 32: 556-559.

Ruel E, E Reither, S Robert, and P Lantz (2010) Neighborhood effects on BMI trends: Examining BMI trajectories for Black \& White women. Health \& Place 16 (2): 191-198.

Satija A; Bhupathiraju, N Shilpa; EB Rimm, E Spiegelman, D Chiuve, E Stephanie, L Borgi Lee, WC Willett, JE Manson, QH Sun, and B Frank (2016) Plant-Based Dietary Patterns and Incidence of Type 2
Diabetes in US Men and Women: Results from Three Prospective Cohort Studies. PLoS.

Schutte JE, EJ Townsend, J Hugg, RF Shoup, RM Malina, and CG Blomqvist (1984) Density of lean body mass is greater in blacks than in whites. J Appl Physiol 56: 1647-1649.

Singh AS, MJ Chinapaw, et al. (2009) Ethnic differences in BMI among Dutch adolescents: What is the role of scrren viewing, active commuting to school \& consumption of soft drinks \& high caloric snacks. Int Journal of Behavioral Nutrition \& Physical Activity 6 (23).

Siri WE (1956) The gross composition of the body. Adv Biol Med Phys 4 (239-279): 513.

Smalley K et al. (1990) Reassesment of body mass indices." American Journal of Clinical Nutrition 52: 405-408.

Smalley KJ, AN Knerr, ZV Kendrick, JA Colliver, and OE Owen (1990) Reassessment of body mass indices. J Clin Nutr 52: 405-8.

Spalding et al. (2008) Dynamics of fat cell turnover in humans. Nature 453:783-787.

Speliotes EK, CJ Willer, SI Berndt, KL Monda, G Thorleifsson, AU Jackson, HL Allen, CM Lindgren, J Luan, and R Mägi (2010) Association analyses of 249,796 individuals reveal 18 new loci associated with body mass index. Nature Genetics 42 (11): 937.

Wagner DR and VH Heyward (2000) Measures of body composition in blacks and whites: a comparative review. Am J Clin Nutr 71 (6): 1392-402. doi: 10.1093/ajcn/71.6.1392.

Walker RS and MJ Hamilton (2008) Life-History Consequences of Density Dependence and the Evolution of the Human Body Size. Current Anthropology 49 (1): 115-155.

Willer, Cristen J, Elizabeth K Speliotes, Ruth JF Loos, Shengxu Li, Cecilia M Lindgren, Iris M Heid, Sonja I Berndt, Amanda L Elliott, Anne U Jackson, and Claudia Lamina. 2009. "Six new loci associated with body mass index highlight a neuronal influence on body weight regulation." Nature Genetics no. 41 (1):25.

Wing MR (2010) The Genetics of Differential Fat Distribution: The Insulin Resistance Atherosclerosis Family Study, Wake Forest University.

Yanagiya T, A Tanabe, A Iida, S Saito, A Sekine, A Takahashi, T Tsunoda, S Kamohara, Y Nakata, and K Kotani (2007) Association of single-nucleotide polymorphisms in MTMR9 gene with obesity. Human Molecular Genetics 16 (24): 3017-3026.

Zonta LA, SD Jayakar, M Bosisio, A Galante, and V Pennettil (1987) Genetic analysis of human obesity in an Italian sample. Human heredity 37 (3): 129-139. 


\section{Johnson et al. (2019) - Supplementary Data}

Suppl. Table A1. Four Component Model Total Body Water (TBW) data in (lbs) for Africans and African Americans.

\begin{tabular}{|c|c|c|c|c|c|c|c|}
\hline \multicolumn{8}{|c|}{$\begin{array}{l}\text { Physiology Data } \\
4 \text { Component Model Total Body Water (TBW) in (lbs) }\end{array}$} \\
\hline ID & Group & $\begin{array}{c}\text { 4C.TBW } \\
(\%)\end{array}$ & Gender & ID & group & $\begin{array}{c}\text { 4C.TBW } \\
(\%)\end{array}$ & Gender \\
\hline P13 & Afr1 & 32 & $\mathrm{M}$ & P32 & AA & 43.6 & $\bar{F}$ \\
\hline P15 & Afr1 & 48 & M & P34 & AA & 48.4 & $\mathrm{M}$ \\
\hline P18 & Afr1 & 34.6 & $\mathrm{~F}$ & P300 & AA & 31 & $\mathrm{~F}$ \\
\hline P17 & Afr1 & 21 & $\mathrm{M}$ & P313 & AA & 24 & $\mathrm{~F}$ \\
\hline P19 & Afr1 & 38.3 & $\mathrm{~F}$ & P325 & AA & 34.1 & $\mathrm{~F}$ \\
\hline P110 & Afr1 & 33.3 & $\mathrm{M}$ & P326 & AA & 31.9 & $\mathrm{~F}$ \\
\hline P111 & Afr1 & 39 & M & P328 & AA & 48.9 & $\mathrm{M}$ \\
\hline P112 & Afr1 & 46.1 & $\mathrm{M}$ & P329 & AA & 36.6 & $\mathrm{M}$ \\
\hline P114 & Afr1 & 31.7 & $\mathrm{M}$ & P332 & AA & 32 & $\mathrm{~F}$ \\
\hline P117 & Afr1 & 32 & M & P333 & AA & 32.4 & $F$ \\
\hline P121 & Afr1 & 32 & $\mathrm{M}$ & P334 & AA & 35.2 & $\mathrm{~F}$ \\
\hline P130 & Afr1 & 23.5 & $\mathrm{M}$ & P36 & AA & 41 & $\mathrm{~F}$ \\
\hline P131 & Afr1 & 41.1 & $\mathrm{M}$ & P338 & AA & 24.1 & $\mathrm{M}$ \\
\hline P135 & Afr1 & 27.9 & $\mathrm{M}$ & P340 & AA & 24.5 & $\mathrm{~F}$ \\
\hline P136 & Afr1 & 35.8 & M & P344 & AA & 27 & $\mathrm{M}$ \\
\hline P141 & Afr1 & 41.3 & $\mathrm{M}$ & P345 & AA & 42 & $\mathrm{~F}$ \\
\hline P144 & Afr1 & 36.3 & M & P347 & AA & 34.7 & $\mathrm{M}$ \\
\hline P146 & Afr1 & 47.7 & M & P349 & AA & 29.4 & $\mathrm{~F}$ \\
\hline P148 & Afr1 & 26.1 & $\mathrm{M}$ & P350 & AA & 32.9 & $\mathrm{M}$ \\
\hline P152 & Afr1 & 30.5 & $\mathrm{M}$ & P351 & AA & 22.6 & $\mathrm{~F}$ \\
\hline P154 & Afr1 & 31.9 & $\mathrm{M}$ & P356 & AA & 24 & $\mathrm{M}$ \\
\hline P155 & Afr1 & 38.7 & $\mathrm{M}$ & P361 & AA & 39.4 & $\mathrm{M}$ \\
\hline P157 & Afr1 & 47.1 & M & 1P3-12 & AA & 47 & $\mathrm{~F}$ \\
\hline P162 & Afr1 & 38.7 & $\mathrm{M}$ & $2 \mathrm{P} 3 \mathrm{~A}-12$ & AA & 53.4 & $\mathrm{~F}$ \\
\hline P164 & Afr1 & 55 & $\mathrm{M}$ & 3P3-12 & AA & 32.7 & $\mathrm{~F}$ \\
\hline 1P1-12 & Afr1 & 51.8 & $\mathrm{M}$ & 4P3-12 & AA & 34.7 & $\mathrm{M}$ \\
\hline $2 \mathrm{P} 1-12$ & Afr1 & 0.36 & $\mathrm{M}$ & $5 \mathrm{P} 3-12$ & AA & 53.1 & $\mathrm{~F}$ \\
\hline $3 \mathrm{P} 1-12$ & Afr1 & 41.3 & $\mathrm{~F}$ & 6P3-12 & AA & 60 & $\mathrm{M}$ \\
\hline 1P2-12 & Afr1 & 61.1 & $\mathrm{M}$ & 7P3-12 & AA & 51 & $\mathrm{~F}$ \\
\hline $2 \mathrm{P} 2-12$ & Afr2 & 63 & $\mathrm{M}$ & 9P3-12 & AA & 35.8 & $\mathrm{M}$ \\
\hline $3 \mathrm{P} 2-12$ & Afr2 & 62.5 & $\mathrm{M}$ & 10P3-12 & AA & 50.7 & $\mathrm{~F}$ \\
\hline $4 \mathrm{P} 2-12$ & Afr2 & 36.2 & $\mathrm{~F}$ & 11P3-12 & AA & 55 & $\mathrm{M}$ \\
\hline P215 & Afr2 & 45.9 & $\mathrm{~F}$ & 12P3-12 & AA & 38.3 & M \\
\hline P216 & Afr2 & 36.8 & $\mathrm{~F}$ & & & & \\
\hline P222 & Afr2 & 32.8 & $\mathrm{~F}$ & & & & \\
\hline P227 & Afr2 & 20.5 & $\mathrm{M}$ & & & & \\
\hline P237 & Afr2 & 45.4 & $\mathrm{~F}$ & & & & \\
\hline P239 & Afr2 & 46.3 & $\mathrm{M}$ & & & & \\
\hline P242 & Afr2 & 32.1 & $\mathrm{~F}$ & & & & \\
\hline P243 & Afr2 & 22.7 & $\mathrm{M}$ & & & & \\
\hline P253 & Afr2 & 34 & $\mathrm{M}$ & & & & \\
\hline P260 & Afr2 & 39.7 & $\mathrm{~F}$ & & & & \\
\hline $\mathrm{P} 263$ & Afr2 & 45.7 & $\mathrm{~F}$ & & & & \\
\hline
\end{tabular}


Suppl. Table A2. African and African American Waist Hip Ratio (WHR).

\begin{tabular}{|c|c|c|c|c|c|c|c|c|c|}
\hline \multicolumn{10}{|c|}{$\begin{array}{l}\text { Physiology Data } \\
\text { African \& African American Waist Hip Ratio (WHR) in (\%) }\end{array}$} \\
\hline ID & Group & WHR & Age & $\begin{array}{c}\text { Gende } \\
\mathbf{r}\end{array}$ & ID & Group & WHR & Age & $\begin{array}{c}\text { Gende } \\
\mathbf{r}\end{array}$ \\
\hline $\mathrm{P} 13$ & Afr & 0.77 & 20 & M & P32 & AA & 0.78 & 23 & $F$ \\
\hline P15 & Afr & 0.81 & 26 & M & P34 & AA & 0.82 & 21 & M \\
\hline P18 & Afr & 0.74 & 32 & $\mathrm{~F}$ & P300 & AA & 0.8 & 33 & $\mathrm{~F}$ \\
\hline P17 & Afr & 0.88 & 38 & $\mathrm{M}$ & P313 & AA & 0.95 & 21 & $\mathrm{~F}$ \\
\hline P19 & Afr & 0.77 & 32 & $\mathrm{~F}$ & P325 & AA & 0.93 & 20 & $\mathrm{~F}$ \\
\hline P110 & Afr & 0.8 & 28 & $\mathrm{M}$ & P326 & AA & 0.8 & 23 & $\mathrm{~F}$ \\
\hline P111 & Afr & 0.82 & 34 & $\mathrm{M}$ & P328 & AA & 0.96 & 21 & $\mathrm{M}$ \\
\hline P112 & Afr & 0.84 & 26 & $\mathrm{M}$ & P329 & AA & 0.86 & 25 & $\mathrm{M}$ \\
\hline P114 & Afr & 0.88 & 36 & $\mathrm{M}$ & P332 & AA & 0.81 & 19 & $\mathrm{~F}$ \\
\hline P117 & Afr & 0.8 & 25 & M & P333 & AA & 0.78 & 22 & $\mathrm{~F}$ \\
\hline P121 & Afr & 0.86 & 27 & $\mathrm{M}$ & P334 & AA & 0.81 & 25 & $\mathrm{~F}$ \\
\hline P130 & Afr & 0.95 & 19 & $\mathrm{M}$ & P36 & AA & 0.89 & 21 & $\mathrm{~F}$ \\
\hline P131 & Afr & 0.79 & 24 & $\mathrm{M}$ & P338 & AA & 0.8 & 25 & $\mathrm{M}$ \\
\hline P135 & Afr & 0.95 & 28 & $\mathrm{M}$ & P340 & AA & 0.85 & 20 & $\mathrm{~F}$ \\
\hline P136 & Afr & 0.83 & 24 & M & P344 & AA & 0.81 & 20 & M \\
\hline P141 & Afr & 0.95 & 21 & $\mathrm{M}$ & P345 & AA & 0.83 & 27 & $\mathrm{~F}$ \\
\hline P144 & Afr & 0.8 & 23 & $\mathrm{M}$ & P347 & AA & 0.82 & 20 & M \\
\hline P146 & Afr & 0.8 & 21 & $\mathrm{M}$ & P349 & AA & 0.75 & 22 & $\mathrm{~F}$ \\
\hline P148 & Afr & 0.89 & 28 & $\mathrm{M}$ & P350 & AA & 0.77 & 25 & $\mathrm{M}$ \\
\hline P152 & Afr & 0.82 & 29 & $\mathrm{M}$ & P351 & AA & 0.77 & 45 & $\mathrm{~F}$ \\
\hline P154 & Afr & 0.94 & 33 & $\mathrm{M}$ & P356 & AA & 0.7 & 22 & $\mathrm{M}$ \\
\hline P155 & Afr & 0.92 & 29 & $\mathrm{M}$ & P361 & AA & 0.89 & 21 & $\mathrm{M}$ \\
\hline P157 & Afr & 0.84 & 37 & $\mathrm{M}$ & 1P3-12 & AA & 0.83 & 21 & $\mathrm{~F}$ \\
\hline P162 & Afr & 0.85 & 24 & $\mathrm{M}$ & 2P3A-12 & AA & 0.76 & 20 & $\mathrm{~F}$ \\
\hline P164 & Afr & 0.83 & 23 & M & 3Р3-12 & AA & 0.76 & 43 & $\mathrm{~F}$ \\
\hline 1P1-12 & Afr & 0.9 & 37 & $\mathrm{M}$ & 4P3-12 & AA & 0.87 & 22 & M \\
\hline $2 \mathrm{P} 1-12$ & Afr & 0.85 & 27 & $\mathrm{M}$ & 5P3-12 & AA & 0.81 & 19 & $\mathrm{~F}$ \\
\hline 3P1-12 & Afr & 0.81 & 27 & $\mathrm{~F}$ & $6 \mathrm{P} 3-12$ & AA & 0.8 & 23 & M \\
\hline 1P2-12 & Afr & 0.89 & 18 & $\mathrm{M}$ & 7P3-12 & AA & 1.06 & 34 & F \\
\hline $2 \mathrm{P} 2-12$ & Afr & 0.83 & 25 & $\mathrm{M}$ & 9P3-12 & AA & 0.8 & 21 & $\mathrm{M}$ \\
\hline $3 \mathrm{P} 2-12$ & Afr & 0.79 & 18 & $\mathrm{M}$ & 10P3-12 & AA & 0.75 & 19 & $\mathrm{~F}$ \\
\hline 4P2-12 & Afr & 0.83 & 19 & $\mathrm{~F}$ & 11P3-12 & AA & 0.98 & 29 & M \\
\hline P215 & Afr & 0.73 & 21 & $\mathrm{~F}$ & $12 \mathrm{P} 3-12$ & AA & 0.83 & 20 & $\mathrm{M}$ \\
\hline P216 & Afr & 0.76 & 18 & $\mathrm{~F}$ & & & & & \\
\hline P222 & Afr & 0.79 & 20 & $\mathrm{~F}$ & & & & & \\
\hline P227 & Afr & 0.88 & 19 & M & & & & & \\
\hline P237 & Afr & 0.84 & 20 & $\mathrm{~F}$ & & & & & \\
\hline P239 & Afr & 0.82 & 19 & $\mathrm{M}$ & & & & & \\
\hline P242 & Afr & 0.78 & 28 & $\mathrm{~F}$ & & & & & \\
\hline P243 & Afr & 0.83 & 20 & $\mathrm{M}$ & & & & & \\
\hline P253 & Afr & 0.81 & 18 & $\mathrm{M}$ & & & & & \\
\hline P260 & Afr & 0.77 & 33 & $\mathrm{~F}$ & & & & & \\
\hline $\mathrm{P} 263$ & Afr & 0.78 & 19 & $\mathrm{~F}$ & & & & & \\
\hline
\end{tabular}




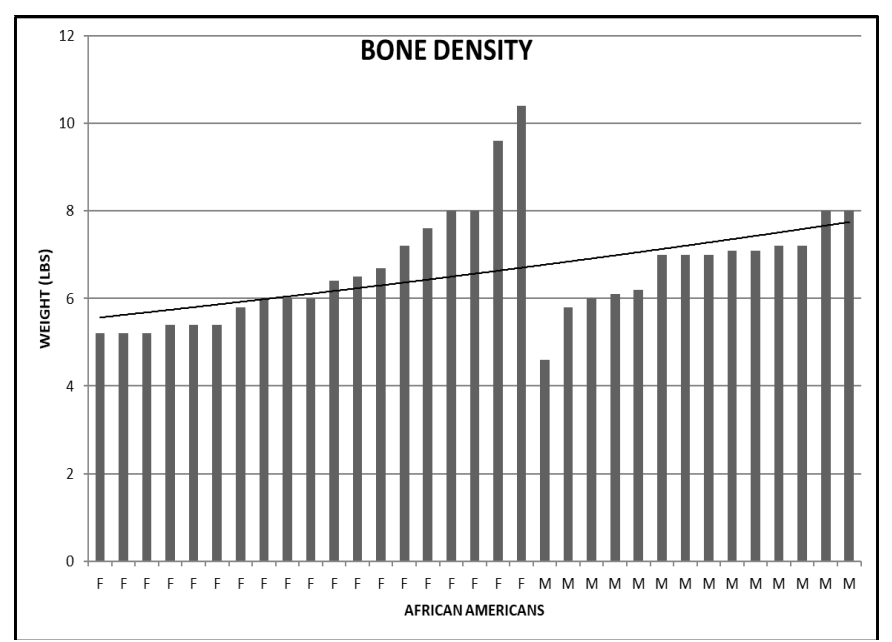

Suppl. Figure A1. African American Bone Density (Db) in (lbs) by Gender.

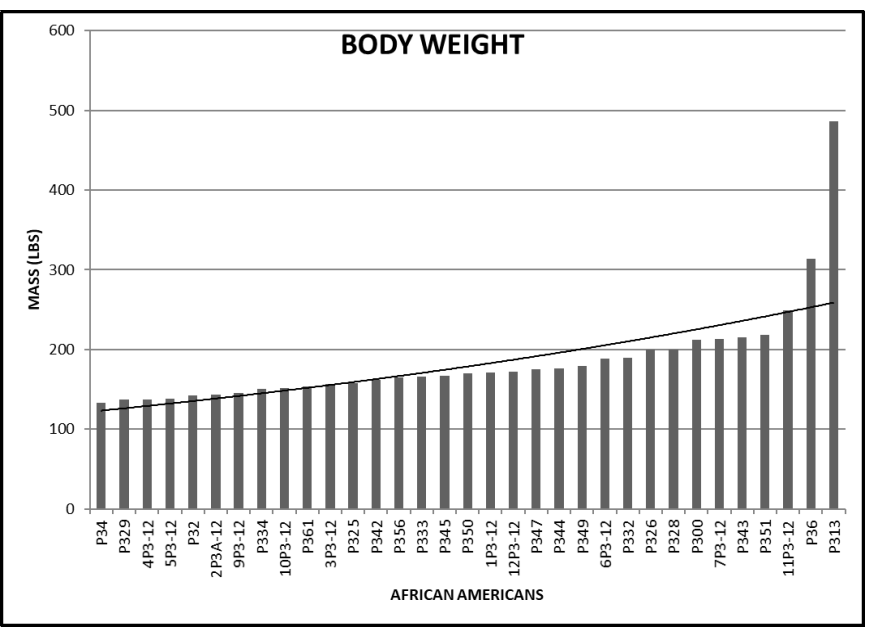

Suppl. Figure A3. African American Body Weight in (lbs).

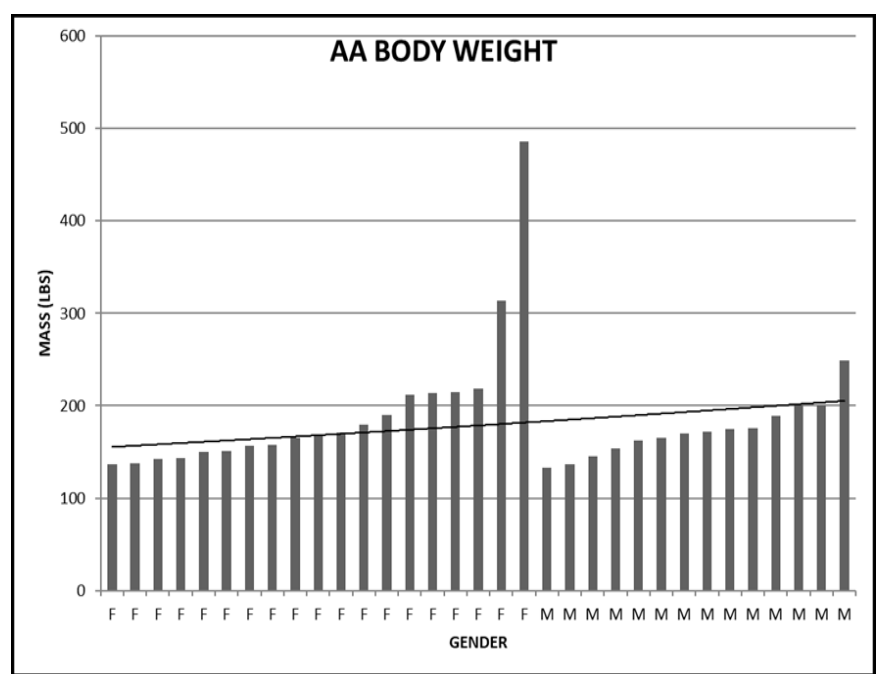

Suppl. Figure A5. African American Body Weight (lbs) by Gender.

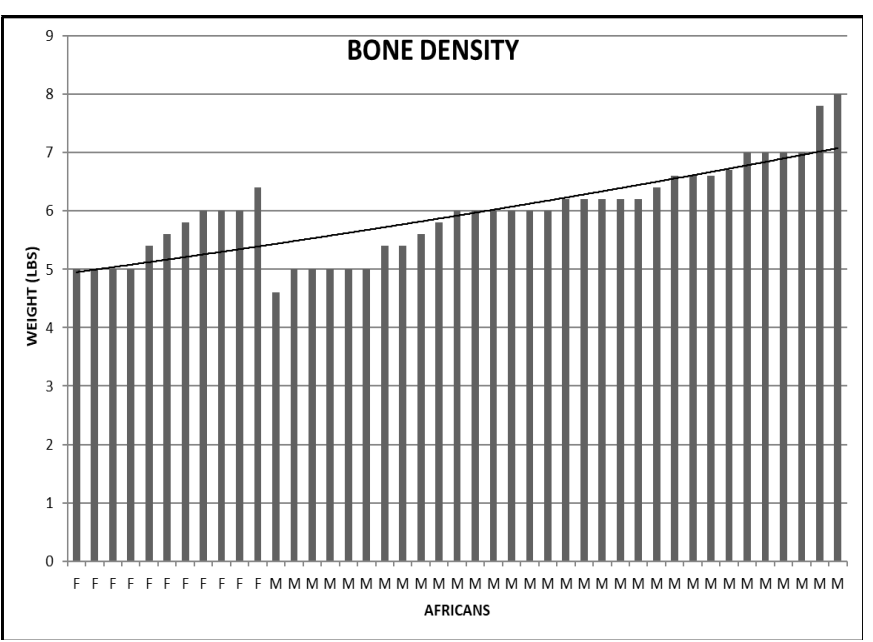

Suppl. Figure A2. African Bone Density (Db) in (lbs) by Gender.

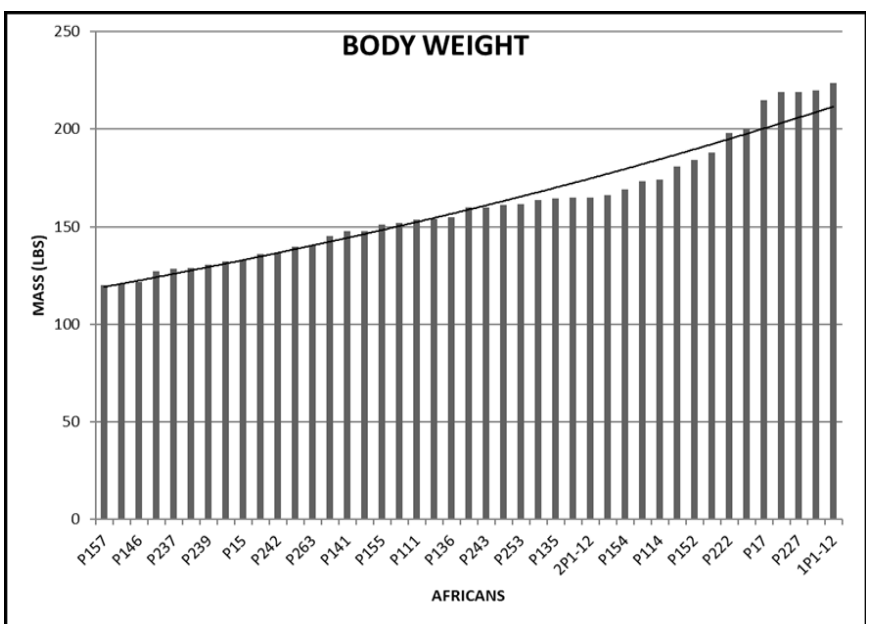

Suppl. Figure A4. African Body Weight in (lbs).

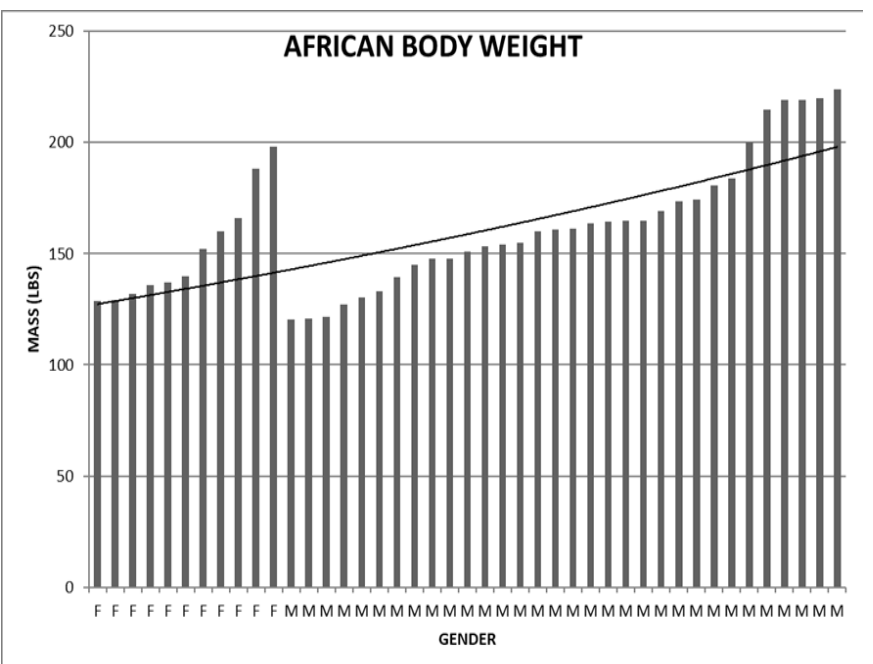

Suppl. Figure A6. African Body Weight (lbs) by Gender. 


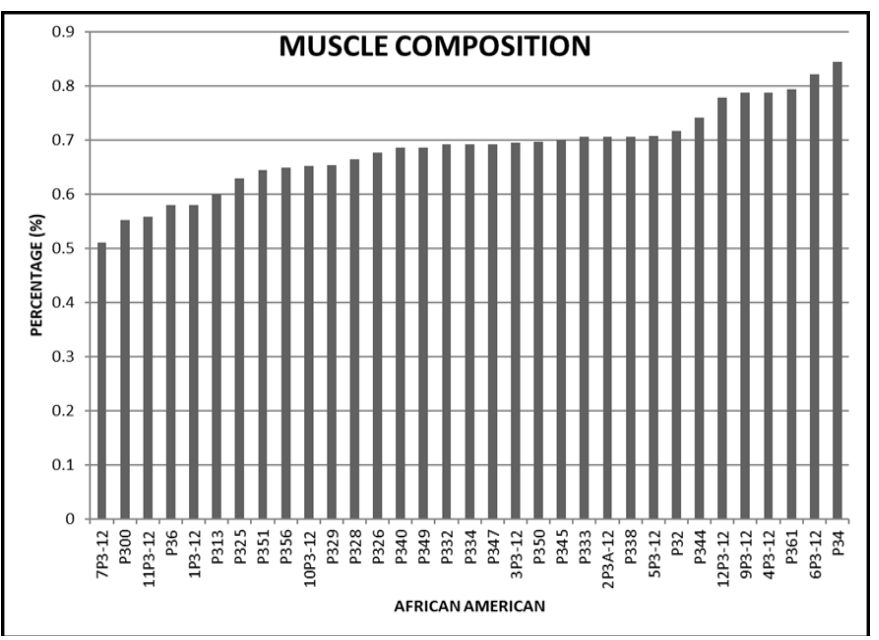

Suppl. Figure A7. African American Muscle Composition (\%). African Americans showed agreement with mesomorphic phenotype, having muscle composition in excess of $60 \%$ of total body mass.

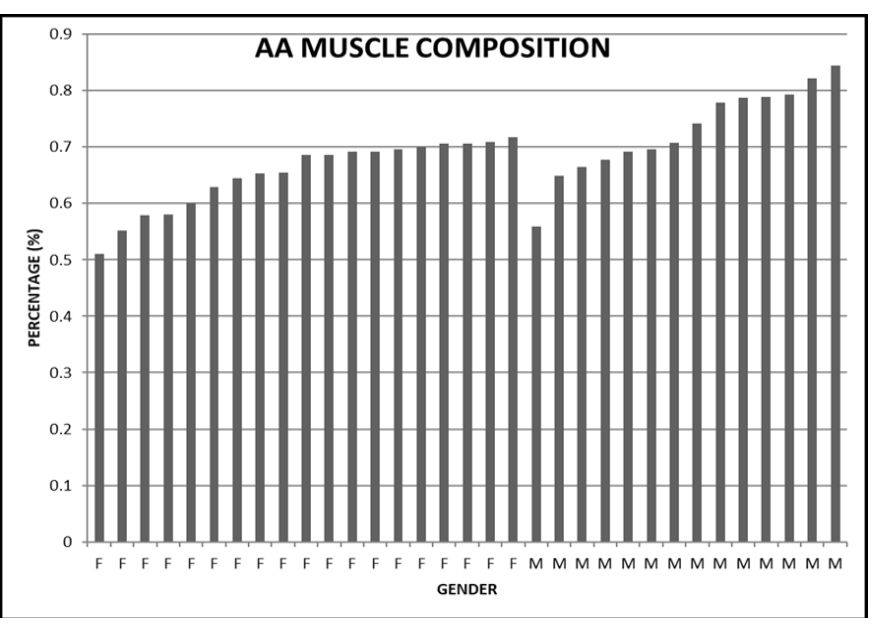

Suppl. Figure A9. African American Male and Female Muscle Composition (\%). AA muscle composition in males and females were found to agree with the mesomorphic phenotype, accounting for $60 \%$ and beyond of total body mass.

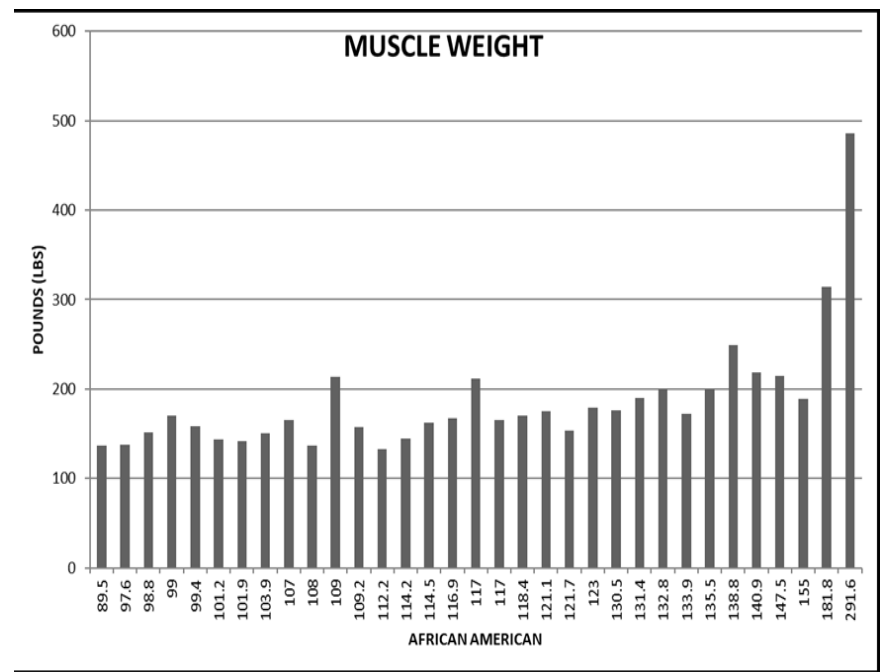

Suppl. Figure A11. African American Muscle Weight (lbs).

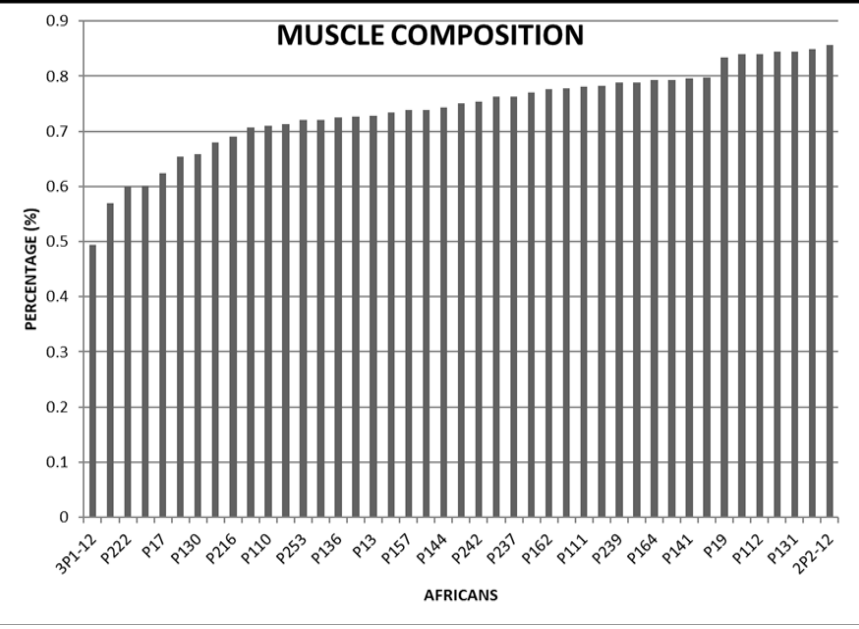

Suppl. Figure A8. African Muscle Composition (\%).African muscle composition was shown to be $60 \%$ and beyond, in agreement with mesomorphic phenotype.

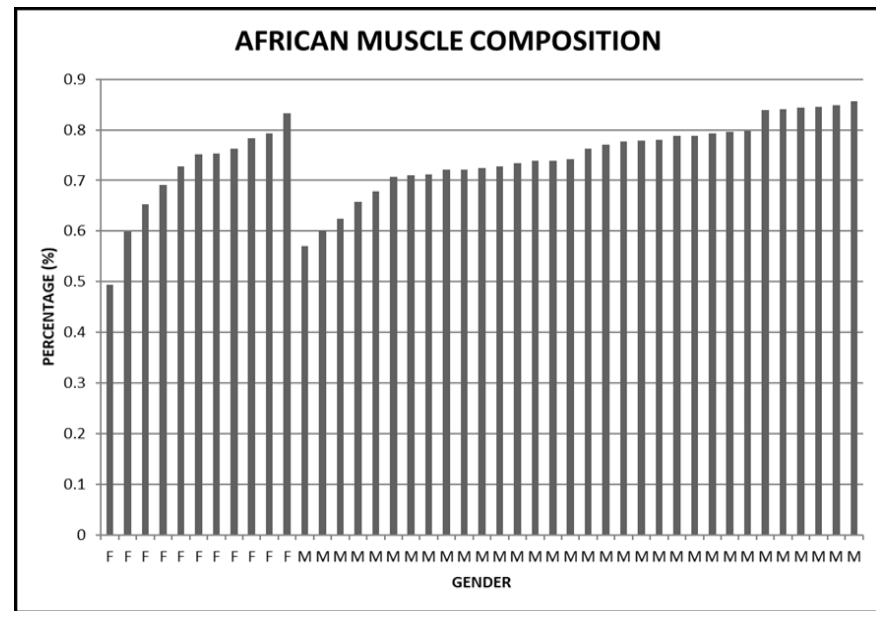

Suppl. Figure A10. African Muscle Composition (\%).

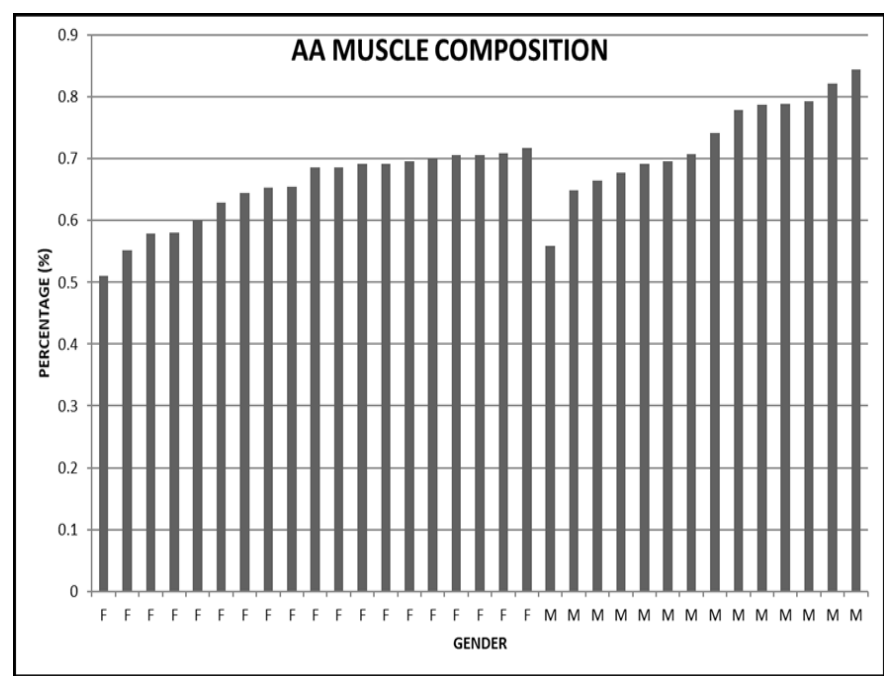

Suppl. Figure A12. African American Muscle Composition (\%) by Gender. 


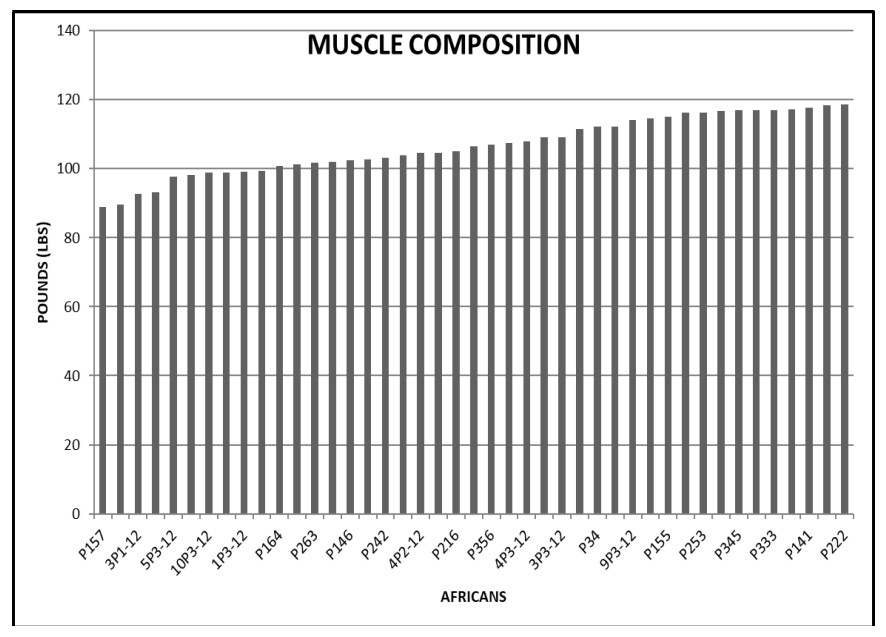

Suppl. Figure A13. African Muscle Composition (lbs).

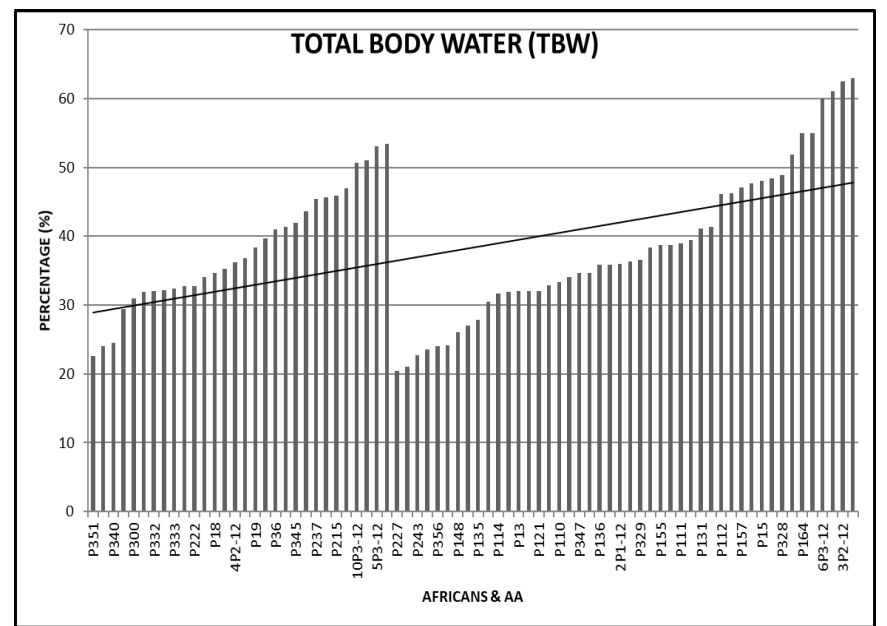

Suppl. Figure A15. African and African American Total Body Water $(\%)$.

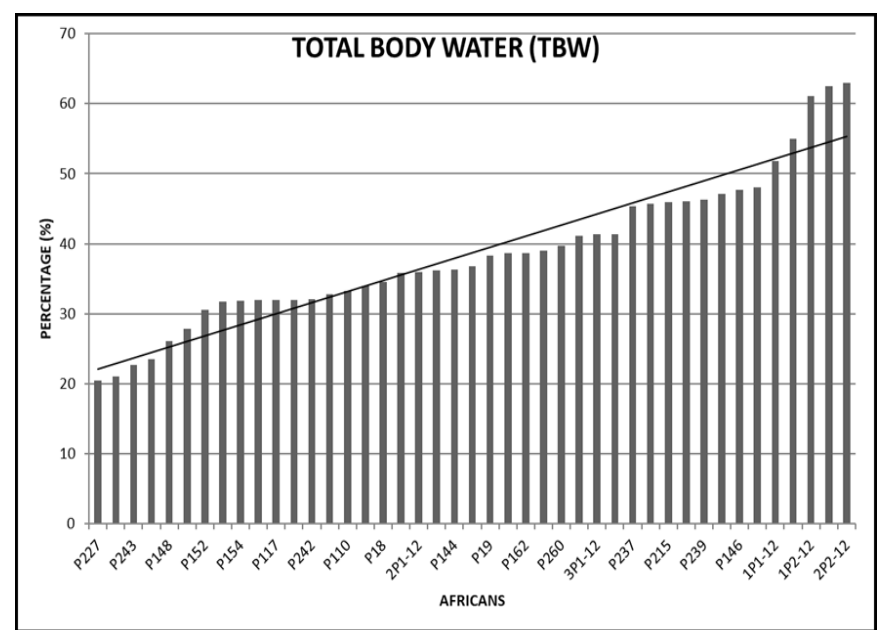

Suppl. Figure A17. African Total Body Water (TBW) by (\%).

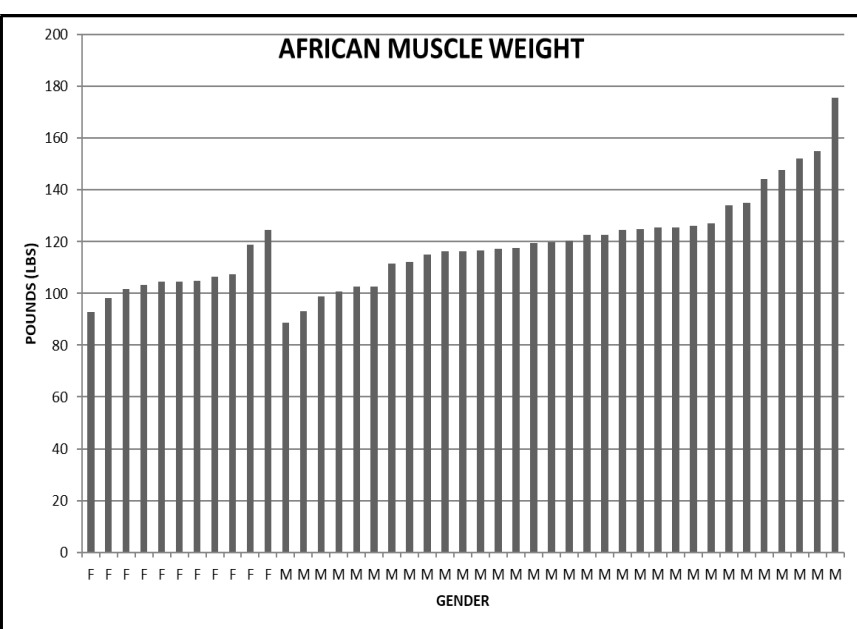

Suppl. Figure A14. African Muscle Weight (lbs) by Gender.

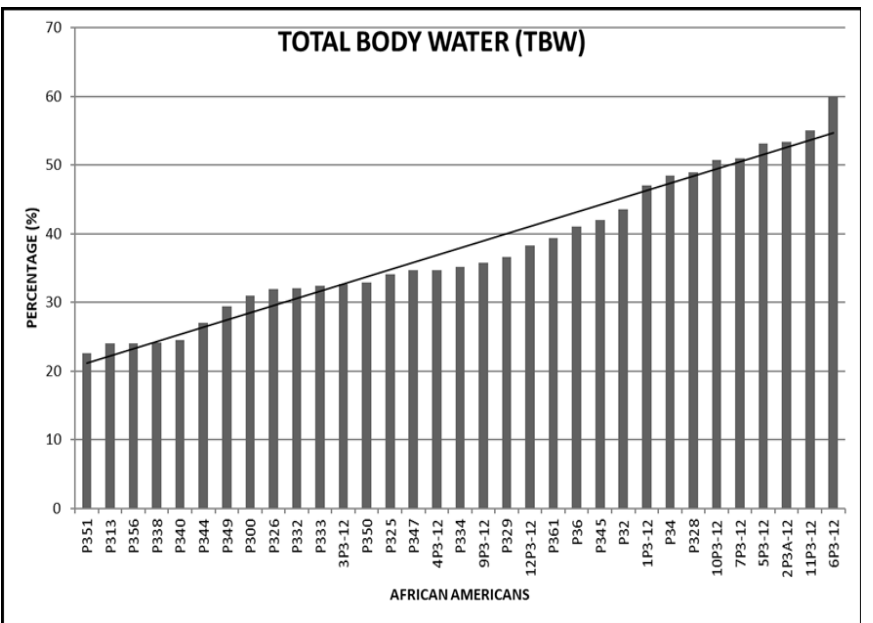

Suppl. Figure A16. African American Total Body Water (\%).

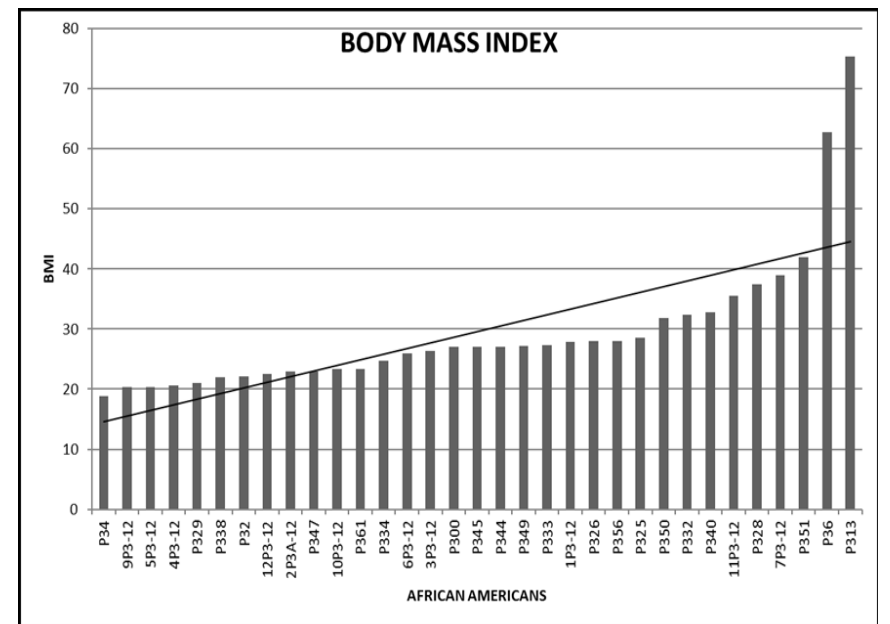

Suppl. Figure A18. African American BMI (\%). 


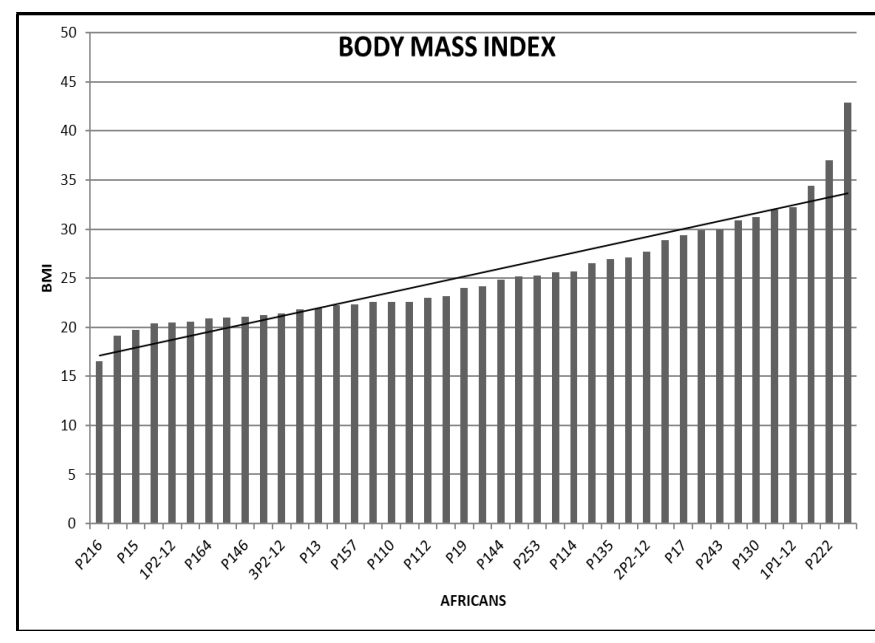

Suppl. Figure A19. African BMI (\%).

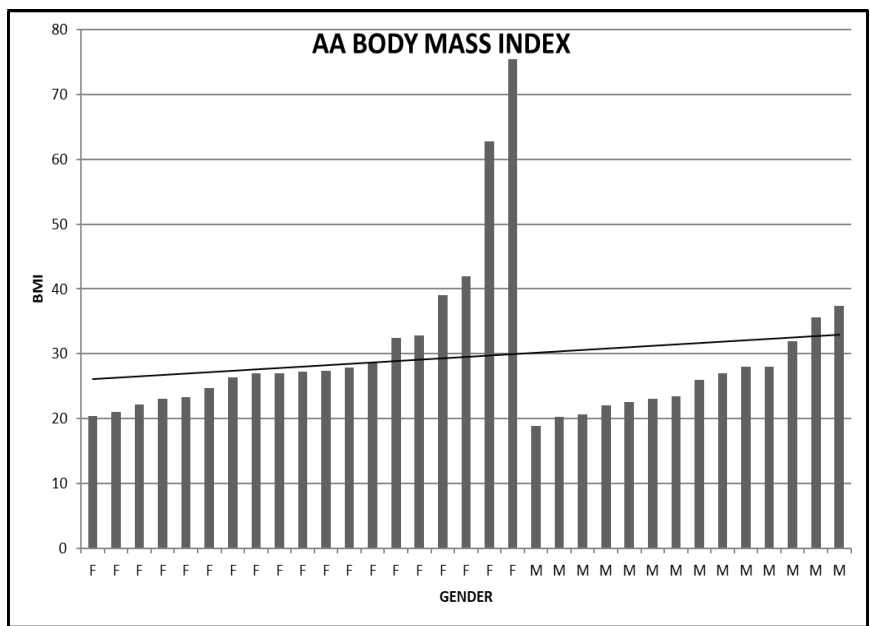

Suppl. Figure A21. African American BMI (\%) by Gender.

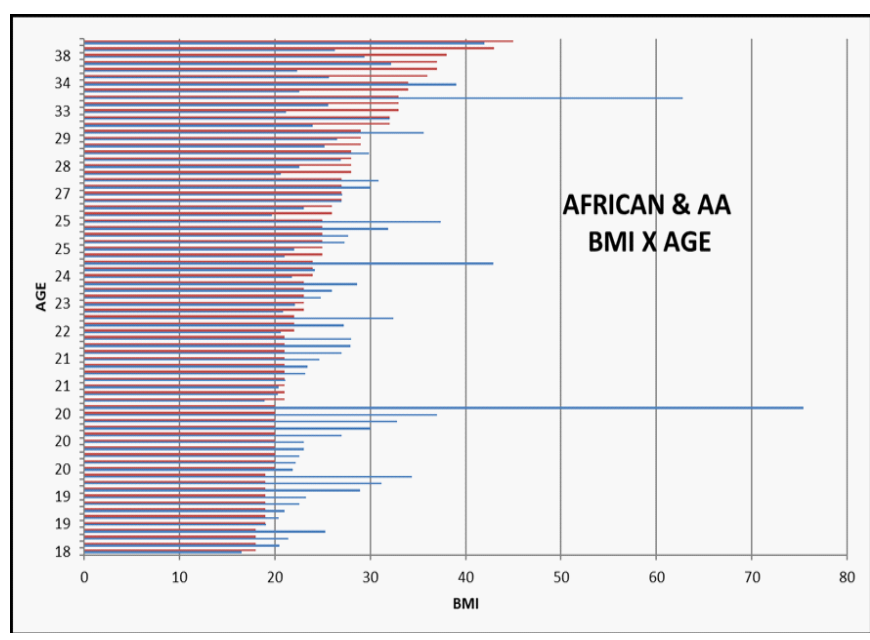

Suppl. Figure A23. African \& African American BMI (\%) by Age.

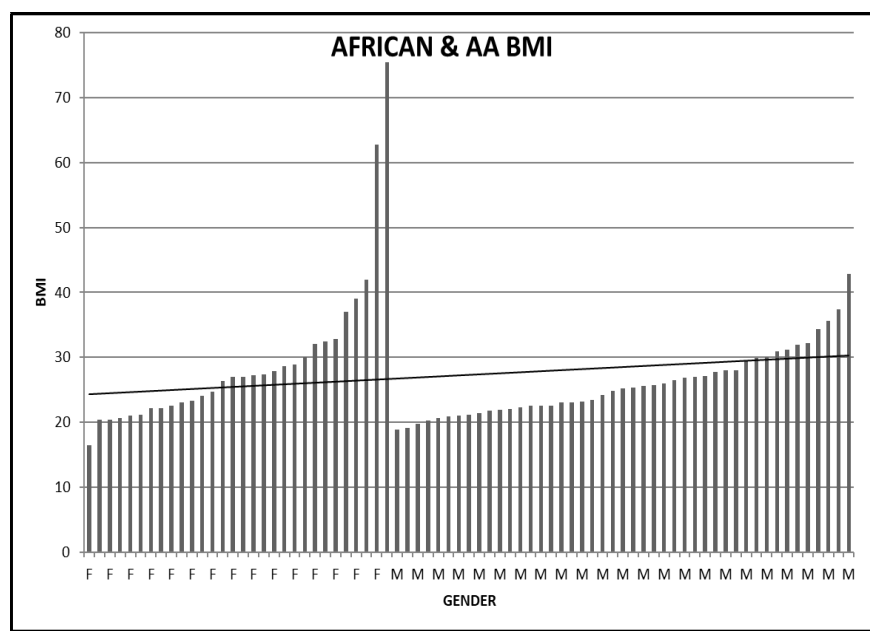

Suppl. Figure A20. African \& African American BMI (\%) by Age.

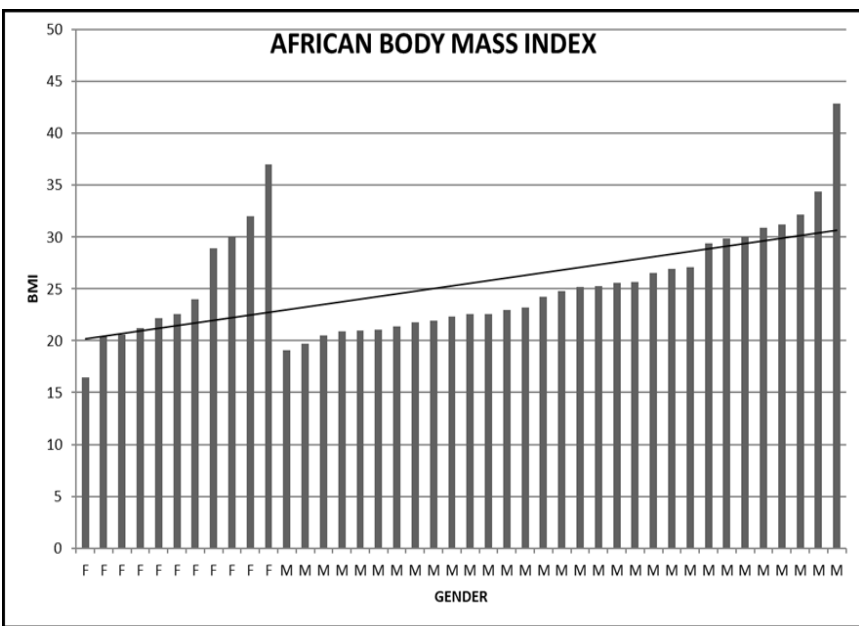

Suppl. Figure A22. African BMI by Gender (\%).

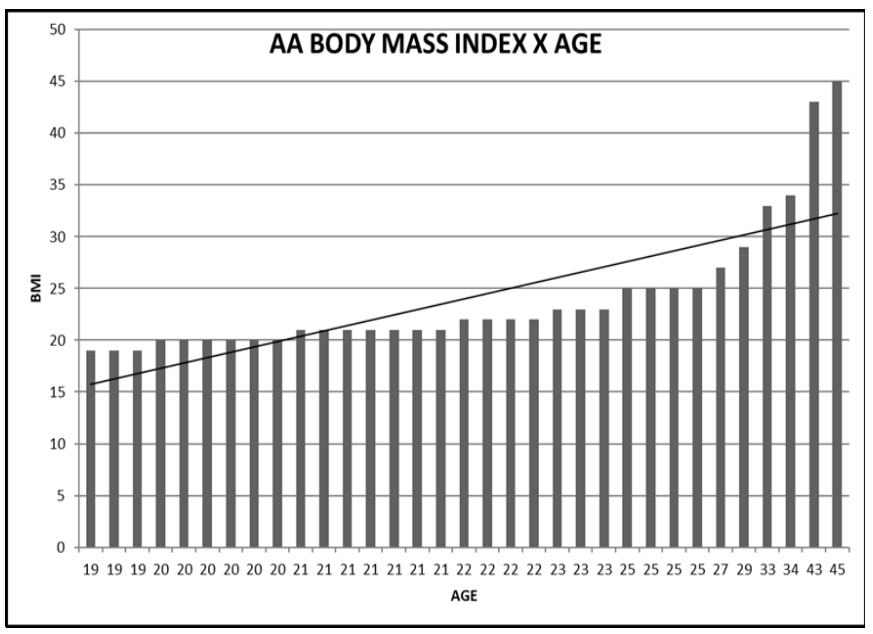

Suppl. Figure A24. African American BMI (\%) by Age. 


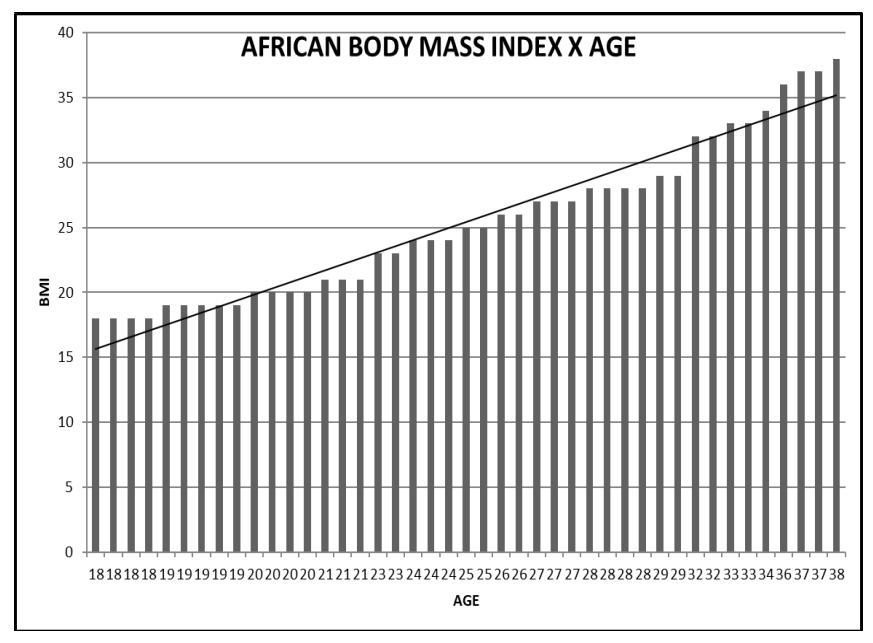

Suppl. Figure A25. African BMI (\%) by Age.

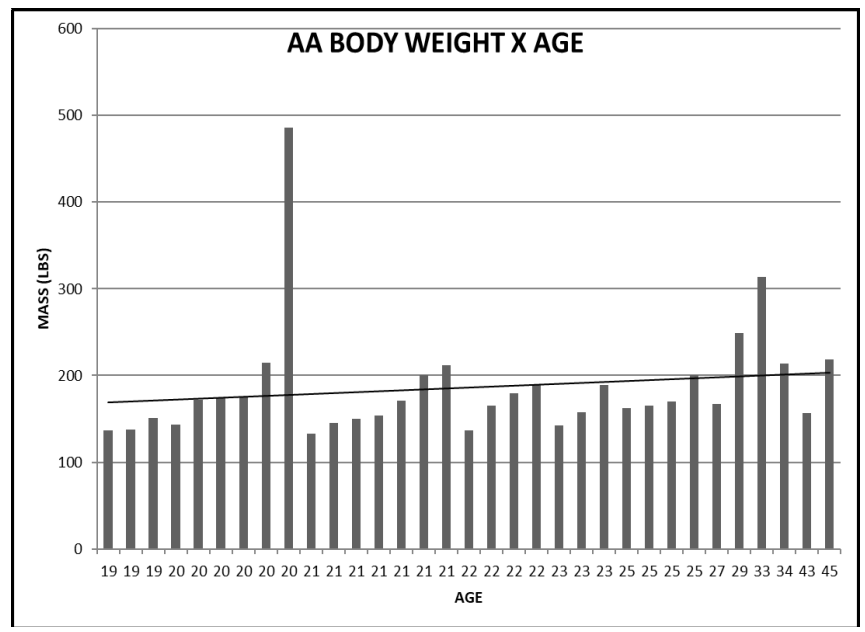

Suppl. Figure A27. African American Body Weight (lbs) by Age.

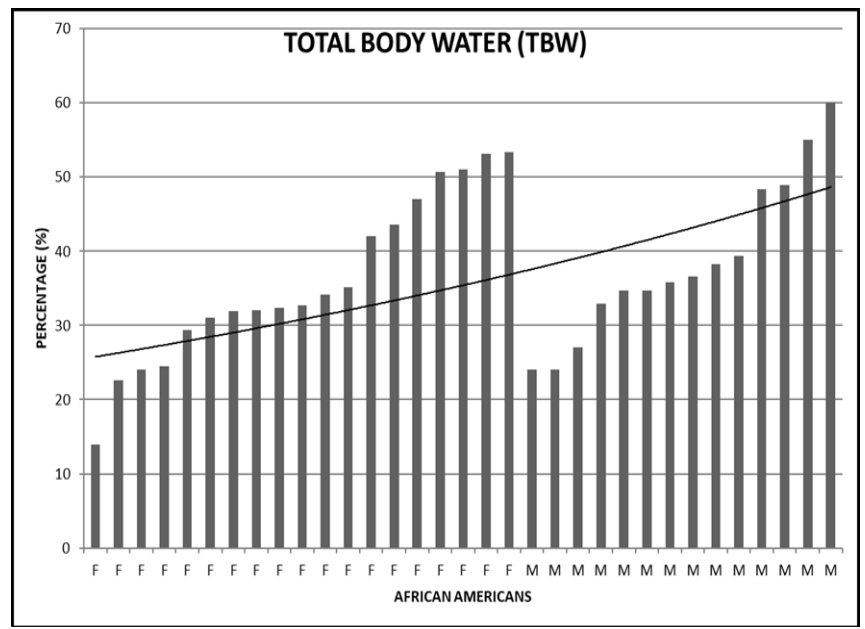

Suppl. Figure A29. African Total Body Water (TBW) by Gender.

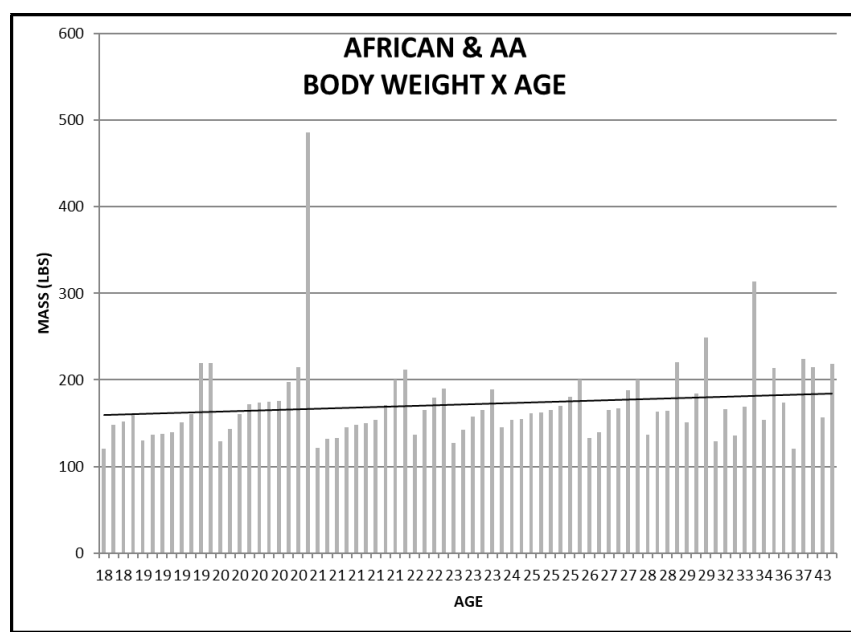

Suppl. Figure A26. African \& African American Body Weight (lbs) by Age.

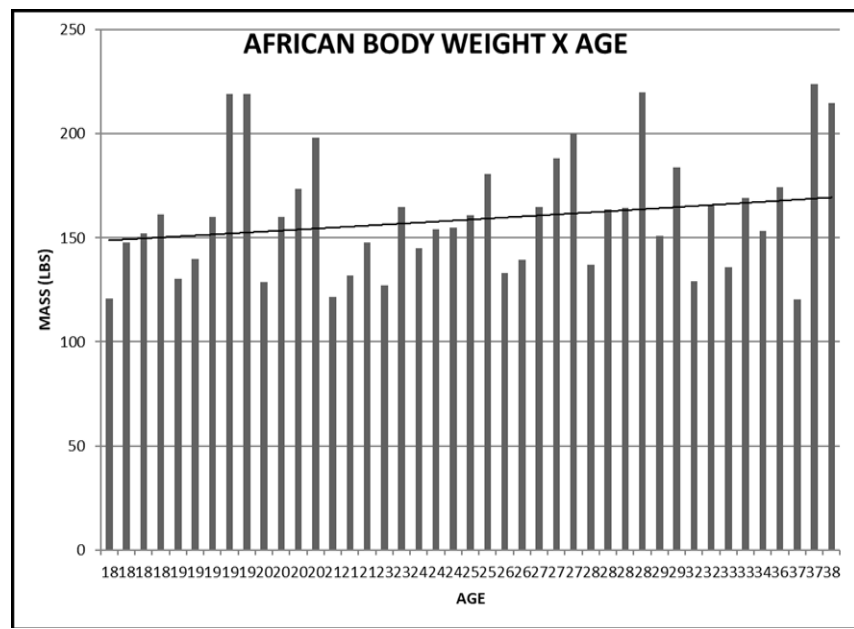

Suppl. Figure A28. African American Total Body Water (\%) by Gender.

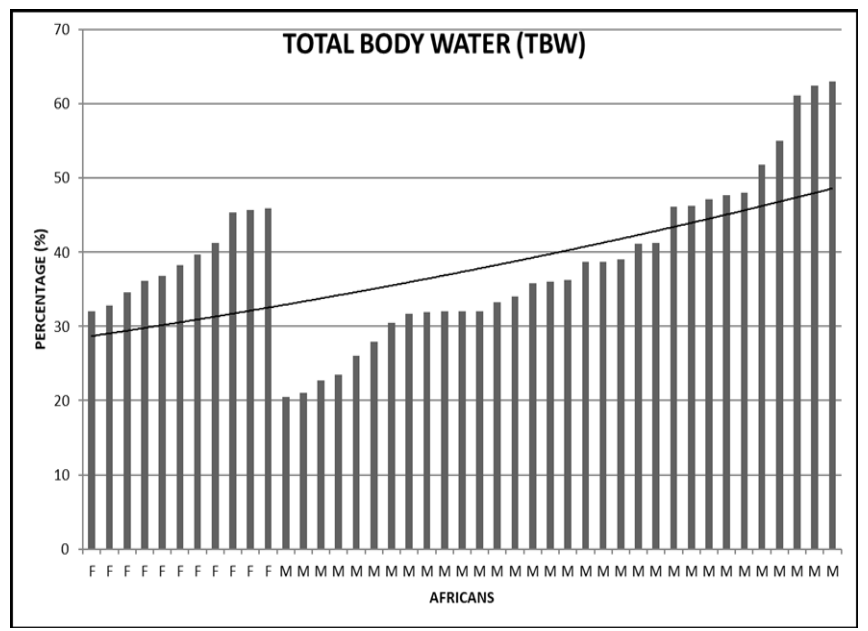

Suppl. Figure A30. African \& African American Waist Hip Ratio (WHR). 


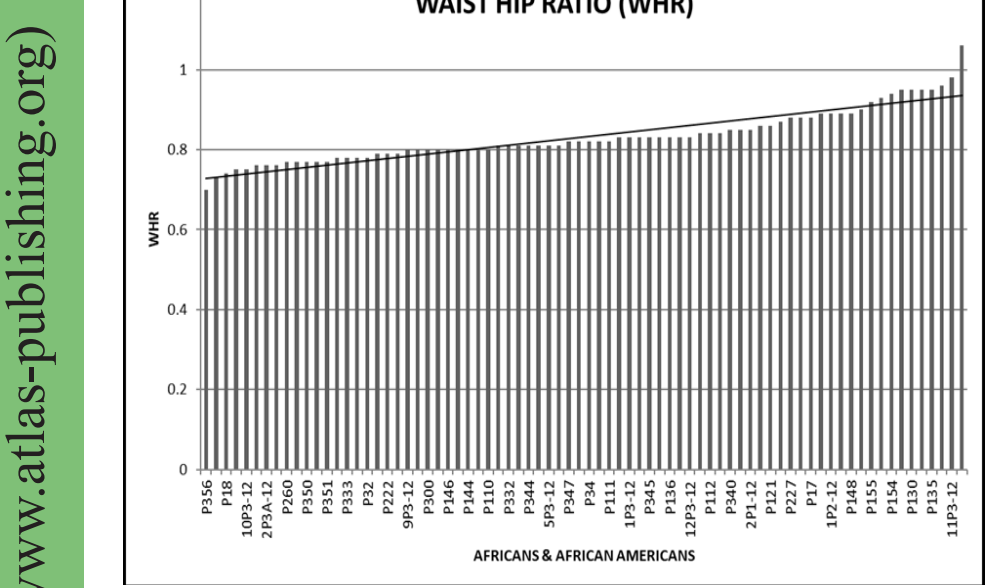

Suppl. Figure A31. African American Waist Hip Ratio WHR (\%).

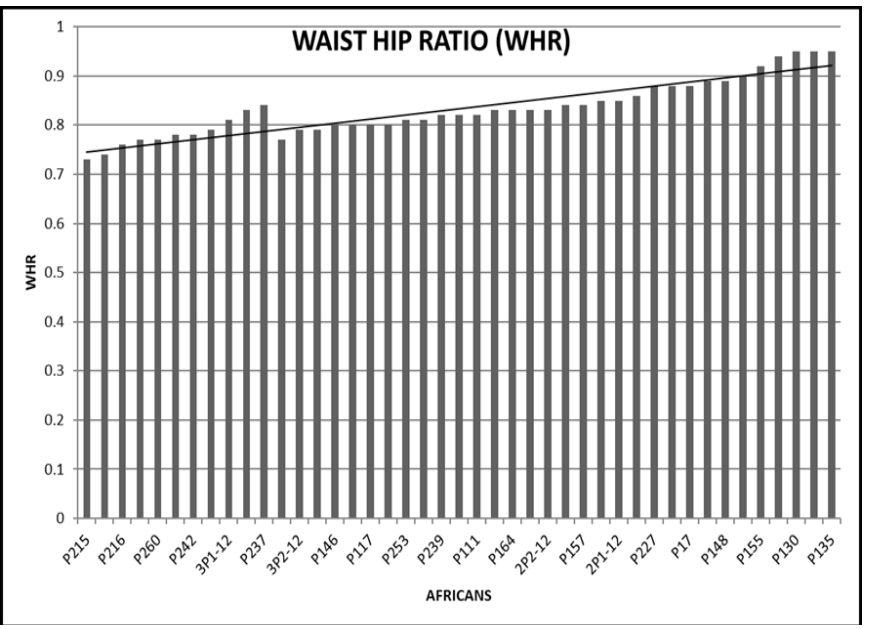

Suppl. Figure A33. African American Waist Hip Ratio -WHR (\%) by Gender.

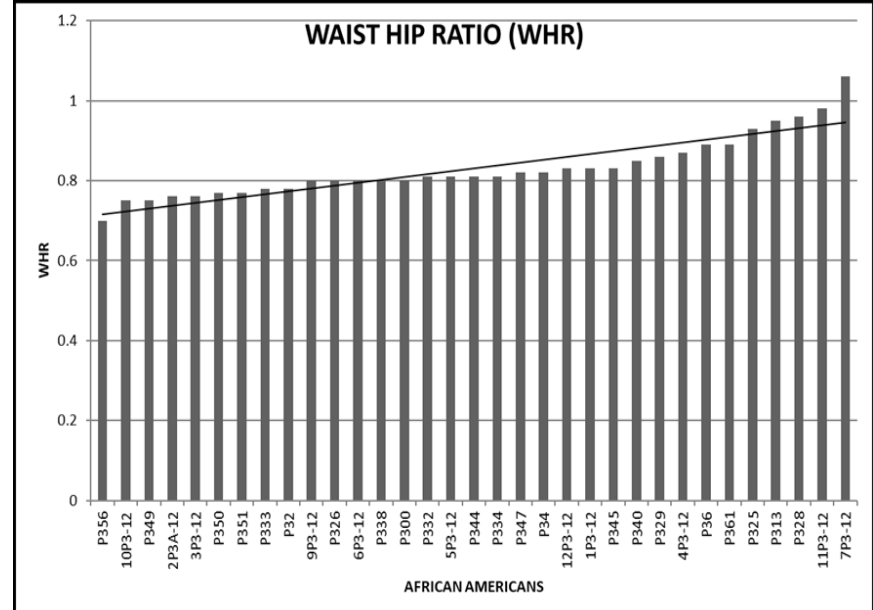

Suppl. Figure A32. African Waist Hip Ratio (\%).

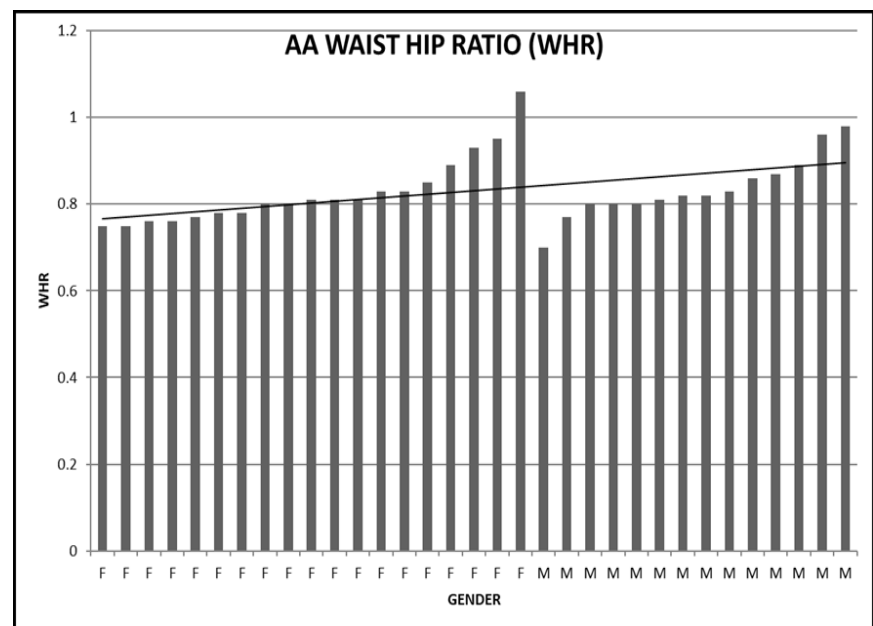

Suppl. Figure A34. African Waist Hip Ratio (\%) by Gender. 